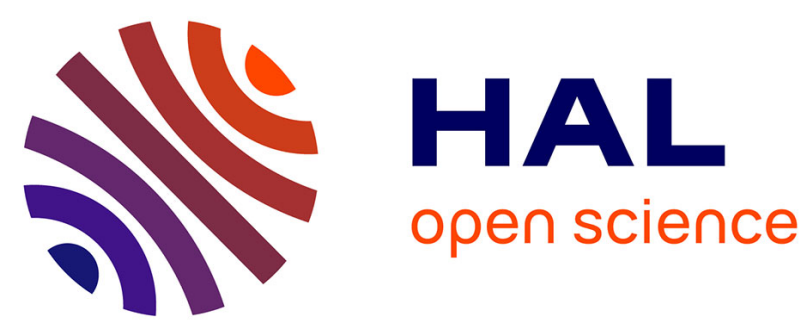

\title{
Ellipticity loss analysis for tangent moduli deduced from a large strain elastic-plastic self-consistent model
}

Gérald Franz, Farid Abed-Meraim, Jean-Paul Lorrain, Tarak Ben Zineb, Xavier Lemoine, Marcel Berveiller

\section{To cite this version:}

Gérald Franz, Farid Abed-Meraim, Jean-Paul Lorrain, Tarak Ben Zineb, Xavier Lemoine, et al.. Ellipticity loss analysis for tangent moduli deduced from a large strain elastic-plastic self-consistent model. International Journal of Plasticity, 2009, 25 (2), pp.205-238. 10.1016/j.ijplas.2008.02.006 . hal-01081905

\section{HAL Id: hal-01081905 \\ https://hal.science/hal-01081905}

Submitted on 12 Nov 2014

HAL is a multi-disciplinary open access archive for the deposit and dissemination of scientific research documents, whether they are published or not. The documents may come from teaching and research institutions in France or abroad, or from public or private research centers.
L'archive ouverte pluridisciplinaire HAL, est destinée au dépôt et à la diffusion de documents scientifiques de niveau recherche, publiés ou non, émanant des établissements d'enseignement et de recherche français ou étrangers, des laboratoires publics ou privés. 


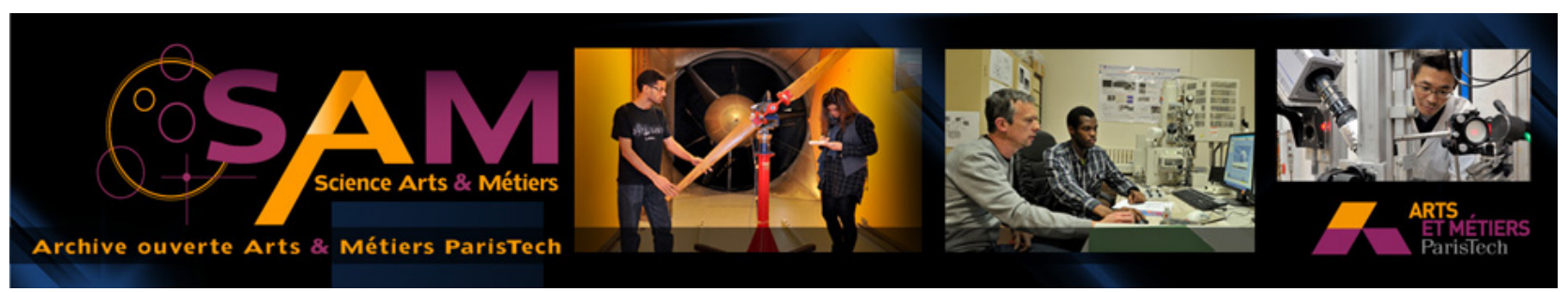

\section{Science Arts \& Métiers (SAM)}

is an open access repository that collects the work of Arts et Métiers ParisTech researchers and makes it freely available over the web where possible.

This is an author-deposited version published in: http://sam.ensam.eu

Handle ID: .http://hdl.handle.net/10985/8874

\section{To cite this version :}

Gérald FRANZ, Farid ABED-MERAIM, Jean-Paul LORRAIN, Tarak BEN ZINEB, Xavier LEMOINE, Marcel BERVEILLER - Ellipticity loss analysis for tangent moduli deduced from a large strain elastic-plastic self-consistent model - International Journal of Plasticity - Vol. 25, $\mathrm{n}^{\circ} 2$, p.205-238- 2009 


\section{Accepted Manuscript}

Ellipticity loss analysis for tangent moduli deduced from a large strain elasticplastic self-consistent model

Gérald Franz, Farid Abed-Meraim, Jean-Paul Lorrain, Tarak Ben Zineb, Xavier Lemoine, Marcel Berveiller

PII:

DOI:

Reference:

To appear in:

Received Date:

Revised Date:

Accepted Date:

$$
\text { S0749-6419(08)00034-X }
$$$$
\text { 10.1016/j.ijplas.2008.02.006 }
$$

$$
\text { INTPLA } 1121
$$

\section{International Journal of Plasticity}

18 July 2007

25 February 2008

27 February 2008

Please cite this article as: Franz, G., Abed-Meraim, F., Lorrain, J-P., Zineb, T.B., Lemoine, X., Berveiller, M., Ellipticity loss analysis for tangent moduli deduced from a large strain elastic-plastic self-consistent model, International Journal of Plasticity (2008), doi: 10.1016/j.ijplas.2008.02.006

This is a PDF file of an unedited manuscript that has been accepted for publication. As a service to our customers we are providing this early version of the manuscript. The manuscript will undergo copyediting, typesetting, and review of the resulting proof before it is published in its final form. Please note that during the production process errors may be discovered which could affect the content, and all legal disclaimers that apply to the journal pertain. 


\title{
Ellipticity loss analysis for tangent moduli deduced
}

\section{from a large strain elastic-plastic self-consistent model}

\author{
Gérald FRANZ ${ }^{\text {, }}$ Farid ABED-MERAIM ${ }^{\text {a,* }}$, Jean-Paul LORRAIN ${ }^{\text {a }}$, Tarak BEN \\ ZINEB $^{b}$, Xavier LEMOINE ${ }^{c}$ and Marcel BERVEILLER ${ }^{a}$
}

${ }^{a}$ Laboratoire de Physique et Mécanique des Matériaux, L.P.M.M., UMR CNRS 7554, 4 rue Augustin Fresnel, 57078 Metz Cedex 3, France

b Laboratoire d’Energétique et de Mécanique Théorique et Appliquée, L.E.M.T.A., UMR CNRS 7563, 2 rue Jean Lamour, 54500 Vandœuvre-lès-Nancy, France

${ }^{\mathrm{c}}$ R\&D Automotive Products, ArcelorMittal Maizières, voie Romaine, B.P. 30320 57283 Maizières-lès-Metz, France

\begin{abstract}
In order to investigate the impact of microstructures and deformation mechanisms on the ductility of materials, the criterion first proposed by Rice is applied to elastic-plastic tangent moduli derived from a large strain micromechanical model combined with a self-consistent scale transition technique. This approach takes into account several microstructural aspects for polycrystalline aggregates: initial and induced textures, dislocation densities as well as softening mechanisms such that the behavior during complex loading paths can be accurately described.

In order to significantly reduce the computing time, a new method drawn from viscoplastic formulations is introduced so that the slip system activity can be efficiently determined. The different aspects of the single crystal hardening (self and latent
\end{abstract}


hardening, dislocation storage and annihilation, mean free path, etc...) are taken into account both by the introduction of dislocation densities per slip system as internal variables and the corresponding evolution equations. Comparisons are made with experimental results for single and dual-phase steels involving linear and complex loading paths. Rice's criterion is then coupled and applied to this constitutive model in order to determine the ellipticity loss of the polycrystalline tangent modulus. This criterion, which does not need any additional "fitting” parameter, is used to build Ellipticity Limit Diagrams (ELDs).

Keywords: Scale transition; ductility; Rice’s criterion; Ellipticity Limit Diagram; plastic instability; bifurcation; loss of ellipticity.

* Corresponding author. Address: ENSAM de Metz, 4 rue Augustin Fresnel, 57078

Metz Cedex 03, France. Tel.: +(33) 3.87.37.54.79; Fax: +(33) 3.87.37.54.70.

E-mail address: farid.abed-meraim@metz.ensam.fr 


\section{Introduction}

During sheet metal forming processes many failure modes may occur. For ductile materials, these modes mostly appear in the inelastic regime and have different origins:

- Buckling and wrinkling have a geometric origin principally; the onset of such mechanisms is strongly dependent on the shape of the part to be formed. They are generally characterized by the occurrence of undesired folds in the final part.

- Localization by shear bands, whose main origin is the material behavior, occurs due to competing hardening and softening mechanisms. In this case, it is clearly observed that plastic strain is concentrated in some narrow bands of the material in the form of shear bands. In uniaxial tension and for isotropic materials, one or two bands oriented at approximately $54^{\circ}$ with respect to the loading direction are generally observed in experiments.

- Necking is due to material softening and geometric changes of the specimen. It starts when the hardening generated by plastic flow becomes too weak to compensate the reduction of the cross section. As a consequence, a non-uniform reduction of the cross section appears.

These mechanisms can sometimes be coupled with damage phenomena that affect the mechanical properties and lead to reduction in ductility (Tang and Tai, 2000; Chow et al., 2001; Chow and Jie, 2004; Menzel et al., 2005; Csontos and Starke, 2005;

Sánchez et al., 2008; Varma et al., 2007). For stretched sheet metals, two forms of necking, namely diffuse and localized necking, may occur. It has been shown that diffuse necking happens prior to localized necking, and it is now well recognized that the maximum allowable straining in sheet metal forming is determined by localized 


\section{ACCEPTED MANUSCRIPT}

necking. As a unified approach that takes into account all these mechanisms seems to be very difficult; the present work focuses on the onset of strain localization due to macroscopic shear band formation. The obtained results for the strain localization predictions will be plotted in terms of curves called Ellipticity Limit Diagrams (ELDs).

The mechanism of shear band formation can be seen as a bifurcation, i.e. several responses of the material (viewed as a system) for a given loading path can exist simultaneously. In order to predict strain localization induced by shear band formation, one of the two following approaches is generally adopted:

- The Marciniak-Kuczynski or M-K (Marciniak and Kuczynski, 1967) approach in which the principal assumption is the presence of an initial defect in the material sheet. This defect is materialized by an oriented band, whose thickness is smaller than that of the rest of the specimen, while the behavior in the two regions is assumed to be identical. The difference in thickness between the two regions grows during loading and the ductility limit is attained when this difference reaches a critical value. In more recent applications of this model, it is the critical value of the major strain rate ratio inside and outside the band that most often marks the limit strains. It is interesting to recall that, in its original version, this model postulated a groove (narrow band) across the width of the sheet (i.e. lying parallel to the minor-strain direction) and was limited to forming limit predictions for positive principal strain ratios - right-hand side of the Forming Limit Diagram (FLD), covering plane strain to equibiaxial tension. This model was subsequently cast into a rigorous framework (Hutchinson and Neale, 1978) by allowing the initial angle of the imperfection band to rotate until a localized neck is detected. A minimization over all initial inclinations of the 
groove provides the limit strains, and in this way the prediction of FLD was extended to its left-hand side, covering uniaxial to plane strain tension. Note also that the original M-K model can be regarded, in a sense, as a complementary approach to Hill's zero-extension criterion (Hill, 1952), which is only applicable to left-hand side of FLD as no zero extension direction exists for positive biaxial stretching. However, since localized necking in biaxial stretching is observed in practice, a pre-existing defect has to be introduced in the M-K model to capture this effect, which may provide some justification to this imperfection theory. Over the last decades, this M-K model has been widely used to simulate FLDs leading to reasonably good agreements when compared with experimental results. However, some additional parameters need to be introduced: initial defect value, critical value. While the initial imperfection value has been shown to strongly influence the results (Baudelet, 1984), no significant effect has been noticed for the critical value. Thus, it is this assumed arbitrary imperfection that has been pointed out as the major drawback of this theory. It is also important to note that the M-K criterion is a two-dimensional one; while ductility problems are usually three-dimensional. Although the M-K model is one of the earliest imperfection theories, its concept is still popular, mainly due to its flexibility, simple use for industrial applications and its applicability to a wide range of material models including rate-independent as well as rate-dependent plasticity.

- The Rudnicki-Rice approach (Rudnicki and Rice, 1975; Rice, 1976) is based on the formation of strain localization bands corresponding to jumps of mechanical fields across interfaces. This theory is related to the ellipticity loss of the elasticplastic tangent modulus. The condition of localization is reached when the first 
zero eigenvalue is seen in the acoustic tensor (Rice, 1976). As opposed to the M$\mathrm{K}$ approach, this theory does not need any additional parameters and can be applied to three-dimensional problems. While M-K analysis has been widely used in the literature, few applications of Rice’s ellipticity loss theory, restricted to plane-stress assumptions and simple behavior models, have been attempted in sheet metal forming for quantifying metals in terms of formability.

In earlier works, the M-K theory has been associated with phenomenological elasticplastic constitutive laws in order to predict FLDs for linear (Barlat, 1989) and complex loading paths (Barata Da Rocha, 1989; Baudelet, 1984; Graf and Hosford, 1983; Aretz, 2007, 2008). The damage effects have also been taken into account (Barlat, 1989; Barata Da Rocha, 1989; Baudelet, 1984). Good results have been obtained but the effect of pre-strain on the FLDs was not well reproduced, mainly due to the considered constitutive law, which was not able to properly describe the material response for complex loading paths.

Ito and co-workers (Ito et al., 2000) have coupled an original constitutive law that takes into account the fact that "the direction of the stress rate affects the direction of the plastic strain rate” (Goya and Ito, 1990) and Hill’s quadratic yield surface with Rice’s criterion to compute FLDs without pre-strain. They showed good agreement between their results and experiments. More recently, Stoughton and Yoon (2005) have investigated the formability of anisotropic materials under non-proportional loading paths. Despite the strain-path dependent nature of the FLDs, they are still frequently considered for sheet metal formability analysis. Alternative use of stress-based FLDs has been suggested by several authors (Arrieux, 1995; Vacher et al., 1998; Stoughton, 2000; Butuc et al., 2006; Simha et al., 2007); the reader can also refer to Stoughton and 
Zhu (2004) for an interesting review of theoretical models of strain-based as well as stress-based FLDs. The path-dependence of the stress-based FLDs in relation with advanced constitutive models and experiments has been recently investigated in detail by Wu et al. (2005a) as well as by Yoshida and co-workers (Yoshida and Kuwabara, 2007; Yoshida et al., 2007b; Yoshida and Suzuki, 2008). Kuwabara (2007) reviewed the advances in experimental techniques, using metal sheets and tubes, and showed experimental evidence to the path-independence of stress-based FLDs.

Following Asaro and Rice (1977) who have introduced the framework of crystal plasticity into Rice's criterion, numerous works have been carried out in order to study the ductility of single crystals (Asaro, 1983; Peirce, 1983; Peirce et al., 1982; Peirce et al., 1983). In these works, it is assumed that only two slip systems can be active. The concept of critical hardening modulus is introduced (Asaro, 1983; Asaro and Rice, 1977; Peirce, 1983; Peirce et al., 1982; Peirce et al., 1983; Rudnicki and Rice, 1975). This modulus is reached, for example, when the hardening slope in the case of uniaxial tension is decreasing towards this critical value. Recently, this concept of critical hardening modulus has been studied in the framework of gradient-dependent elasticplastic models (Vrech and Etse, 2006). Necking prediction has also been analyzed using finite element method (Peirce et al., 1982; Peirce et al., 1983) for a plate with an initial imperfection. The thickness and orientation of the localization band are found to be strongly dependent on the initial defect. Although this intrinsic criterion can be applied to study FLDs, no scale transition scheme has been used to derive the behavior of polycrystalline aggregates and thus no FLD predictions have been attempted. In more recent approaches, single crystal plasticity behavior models have been incorporated into finite element method to numerically simulate localized necking (Viatkina et al., 2005; 
Wu et al., 2007; Zhang et al., 2008). Mesh sensitivity is another important drawback of such FE-based formability analyses (Harewood and McHugh, 2006). It is shown, however, that this mesh sensitivity can be removed when using non-local crystal plasticity models such as those considered in Borg (2007).

It has also been shown (Horstemeyer, 2000) that the adopted elastic-plastic constitutive laws have a very strong impact on the FLD predictions. The effect of complex strain paths is, most of the time, not taken into account in phenomenological constitutive laws and it is the same for the FLD simulations. The use of advanced, more predictive constitutive laws seems to be an interesting way to enhance FLD predictions. An attempt to do this can be found in the work of Hiwatashi et al. (1998) who applied an advanced, anisotropic constitutive model based on texture and dislocation structure to predict FLDs, and thus allowing the effect of strain-path changes to be investigated.

Recently, some works have been done by coupling a constitutive law derived from a micro-macro approach with a ductility limit criterion. Boudeau and co-workers (Boudeau et al., 1998) have coupled a micromechanical local law together with the Taylor-Lin scale transition scheme within a small perturbation analysis in order to compute FLDs with or without pre-strain. They obtained good qualitative results. More recent works (Zhou and Neale, 1995; Inal et al., 2005; Wu et al., 2005b) have used a micromechanical local law where slip rates were governed by a power-law relation and hardening by a saturating law. The scale transition was performed by the Taylor-Lin scheme. This micro-macro model has been combined with the M-K analysis in order to simulate FLDs without pre-strain in the cases of B.C.C. and F.C.C. polycrystals with or without texture evolution. In the first contribution (Zhou and Neale, 1995), the imperfection groove, on which the M-K approach is based, was restricted to be normal 
to the major principal stretch direction, while no restriction was imposed in the subsequent analyses (Inal et al., 2005; Wu et al., 2005b). They have demonstrated that the obtained results were in qualitative agreement with expectations. Taylor-based micromechanical models have also been used in the framework of the M-K analysis (Kuroda and Tvergaard, 2000; Knockaert et al., 2002; Yoshida et al., 2007a) for the prediction of FLDs. Very recently (Signorelli et al., 2008), some advances in the FLD analysis have been made by considering a rate-dependent polycrystalline self-consistent plasticity model in conjunction with the M-K approach; while previous M-K applications were restricted to the full-constraint Taylor model.

Therefore, it appears that Rice's criterion is able to provide reliable predictions, sensitive to intrinsic material characteristics, behavior, and changing loading paths under the condition that the behavior is accurately described. This is understandable, due to the fact that several mechanisms can contribute to macroscopic softening of the material and then to strain localization. These mechanisms are:

- Effects related to the spin of stressed lattices leading to convective terms in the constitutive equations. These terms are taken into account within the large strain framework by the use of an objective derivative. The influence of crystallographic structure is very important, for instance, in the case of two single crystals of B.C.C. and F.C.C. structures with the same initial orientation, their crystallographic evolutions are in opposite sense.

- Softening associated with the possibility of multislip in order to accommodate a given strain. Generally crystals with hexagonal structure are less ductile than cubic ones because the number of possible active slip systems is smaller for hexagonal crystals than cubic ones. This effect can be encountered in the work 
of Iwakuma and Nemat-Nasser (1984) who have combined a sensitive micromacro approach in order to model the behavior and the ductility loss using Rice’s criterion. However, by imposing only two given active slip systems, the ductility limit is reached for unusually small strains. By allowing more than two systems to be active by multislip, crystalline plasticity could be very interesting and able to take into account this effect.

- Softening due to enhanced dislocation annihilation when their densities reach significant values. This mechanism can be taken into account by considering the dislocation density for each slip system as an internal variable and by modeling the hardening by means of the evolution of dislocation densities (Berveiller and Zaoui, 1979; Franciosi, 1984; Tabourot, 1992; Hoc et al., 2001).

- Crystallographic softening (or hardening) due to the rotation of crystalline lattice and second order internal stresses.

- Textural softening and induced anisotropy of the polycrystal (crystallographic as well as morphological).

- Possibly, thermomechanical coupling related to intrinsic dissipation and to temperature increase.

An a priori evaluation of the respective influence of all these mechanisms for any loading path appears to be difficult but may be done in the future by using recently developed advanced micromechanical tools. The main objective here is to predict the ductility loss with maximum accuracy by taking into account the influence of changing loading paths and mechanical properties with the aim of designing new materials. For this purpose, Rice’s criterion will be coupled with a constitutive law derived from a large strain micromechanical approach and a self-consistent scale transition scheme. 
The present paper is divided into three parts:

In the first part, the single crystal behavior is modeled within the large strain crystal plasticity framework. The behavior is assumed to be elastic-plastic and a new method to efficiently determine the set of active slip systems is proposed. This method drawn from the viscoplasticity framework - without the need of introducing rate-sensitivity - allows up to $95 \%$ of computing time to be saved while maintaining the same predictions as those of more classical methods. The hardening matrix is reformulated considering the mean dislocation density as an internal variable for each slip system. Latent hardening and dislocation annihilation are taken into account as well as their mean free path through the definition of this matrix.

In the second part, the macroscopic behavior is derived from the local behavior within the framework of Hill's self-consistent scheme (Hill, 1965a,b). At this stage, textural and morphological evolutions are introduced in the model. In order to allow comparisons between experiments and simulations, material parameters need to be identified: four parameters for single phase materials and six parameters for dual-phase materials. They are identified by using two loading paths and the method of identification is explained in detail. Then, comparisons for several sequential rheological tests are presented for a single-phase and a dual-phase steel.

In the third part, Rice's criterion is combined with the macroscopic constitutive law in order to detect the ductility limit. It is worth noting that no additional parameters are introduced at this level; all parameters were identified when the stress-strain curves were analyzed. The ductility limit predictions obtained by this criterion are discussed and synthesized using the concept of Ellipticity Limit Diagrams ELDs. 


\section{Single crystal modeling}

In this part, the single crystal behavior is modeled within the large strain framework in which elastic strains are assumed to remain small. The local incremental elasticplastic constitutive law is defined by means of the tangent modulus $\mathbf{I}$ relating the nominal stress rate $\dot{\mathbf{n}}$ to the velocity gradient $\mathbf{g}$ as:

$$
\dot{\mathbf{n}}=\mathbf{l}: \mathbf{g}
$$

The developed model is based on previous works of Iwakuma and Nemat-Nasser (1984). Some improvements have also been introduced: more than two slip systems can be active, a new criterion for the determination of the set of active slip systems and a new formula for the slip rate calculation are proposed. These improvements reduce considerably the computing time and lead to an advanced, physically based model. Moreover, the hardening matrix is derived from the expression of dislocation densities on the crystallographic slip systems and their evolution laws taking into account storage and annihilation of dislocations. As a consequence, the mean dislocation densities acting on the slip systems are taken as internal variables.

\subsection{Classical framework}

The velocity gradient $\mathbf{g}$, due to the application of macroscopic boundary conditions and loading, induces a crystallographic slip $\dot{\gamma}$ associated with the motion of dislocations (commonly called plastic distortion) and an elastic accommodation with strain and lattice distortion. This velocity gradient $\mathbf{g}=\frac{\partial \mathbf{v}}{\partial \mathbf{x}}$ is additionally decomposed into a symmetric part $\mathbf{d}$ corresponding to the total strain rate and a skew-symmetric one 
w corresponding to the total rotation rate. These two parts can also be divided into an elastic part with the superscript ${ }^{e}$ and a plastic one with the superscript ${ }^{p}$ :

$$
\left\{\begin{array}{l}
\mathbf{g}=\mathbf{d}+\mathbf{w} \\
\mathbf{d}=\mathbf{d}^{e}+\mathbf{d}^{p} \\
\mathbf{w}=\mathbf{W}^{e}+\mathbf{w}^{p}
\end{array}\right.
$$

The plastic parts of $\mathbf{d}$ and $\mathbf{w}$ are expressed as:

$$
\left\{\begin{array}{l}
\mathbf{d}^{p}=\sum_{h} \mathbf{R}^{h} \dot{\gamma}^{h} \\
\mathbf{w}^{p}=\sum_{h} \mathbf{S}^{h} \dot{\gamma}^{h}
\end{array}\right.
$$

where $\mathbf{R}^{h}$ and $\mathbf{S}^{h}$ are the symmetric and skew-symmetric part, respectively, of the Schmid tensor of the slip system $h$. For B.C.C. materials, tensors $\mathbf{R}$ and $\mathbf{S}$ are defined from the unit normal $\overrightarrow{\mathbf{n}}$ to the slip plane and the unit vector $\overrightarrow{\mathbf{m}}$ in the slip direction as:

$$
\left\{\begin{array}{l}
\mathbf{R}=\frac{1}{2}(\overrightarrow{\mathbf{m}} \otimes \overrightarrow{\mathbf{n}}+\overrightarrow{\mathbf{n}} \otimes \overrightarrow{\mathbf{m}}) \\
\mathbf{S}=\frac{1}{2}(\overrightarrow{\mathbf{m}} \otimes \overrightarrow{\mathbf{n}}-\overrightarrow{\mathbf{n}} \otimes \overrightarrow{\mathbf{m}})
\end{array}\right.
$$

where $\otimes$ designates the tensorial product. In Table 1 , the slip systems for B.C.C.

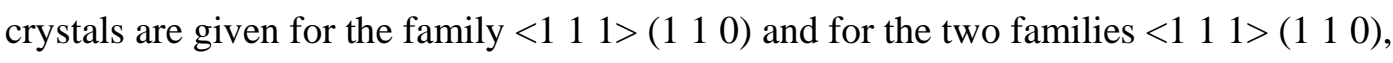
$<111>\left(\begin{array}{lll}1 & 1 & 2\end{array}\right)$.

Elasticity is assumed to be linear and Hooke's law takes the form:

$$
\hat{\sigma}=\mathbf{C}: \mathbf{d}^{e}-\sigma \operatorname{tr}(\mathbf{d})
$$

where $\mathbf{C}$ is the fourth-order tensor of the elastic constants, $\operatorname{tr}(\cdot)$ denotes the trace operator, $\sigma$ is the Cauchy stress and $\hat{\sigma}$ is the lattice co-rotational derivative given by:

$$
\hat{\sigma}=\dot{\sigma}+\sigma \cdot \mathbf{w}^{e}-\mathbf{w}^{e} \cdot \sigma
$$


This derivative follows the crystallographic rotation during loading. The relation between Cauchy's co-rotational derivative and the nominal stress rate is:

$$
\hat{\sigma}=\dot{\mathbf{n}}+\mathbf{w}^{p} \cdot \sigma-\sigma \cdot \mathbf{w}^{p}-\sigma \operatorname{tr}(\mathbf{d})+\mathbf{d} \cdot \sigma+\sigma \cdot \mathbf{w}
$$

For a plastic-type behavior, the definition of the plastic yield or critical shear stress leads to the following flow rule for a given slip system g:

$$
\left\{\begin{array}{l}
\tau^{g}<\tau_{c}^{g} \Rightarrow \dot{\gamma}^{g}=0 \\
\tau^{g}=\tau_{c}^{g} \text { and } \dot{\tau}^{g} \leq 0 \Rightarrow \dot{\gamma}^{g}=0 \\
\tau^{g}=\tau_{c}^{g} \text { and } \dot{\tau}^{g}>0 \Rightarrow \dot{\gamma}^{g} \geq 0
\end{array}\right.
$$

where $\tau^{g}$ and $\tau_{c}^{g}$ are the resolved shear stress acting on the system $g$ and the corresponding critical shear stress, respectively. Eq. (8) can be written in Kuhn-Tucker form, i.e. $\left\{\left(\tau^{g}-\tau_{c}^{g}\right) \leq 0, \dot{\gamma}^{g} \geq 0\right.$ and $\left.\dot{\gamma}^{g}\left(\tau^{g}-\tau_{c}^{g}\right)=0\right\}$, which leads to the necessary condition of consistency $\left(\dot{\tau}^{g}=\dot{\tau}_{c}^{g}\right)$ for $\dot{\gamma}^{g}>0$. It has been shown that several solutions for the set of Eqs. (8) can exist at the same time (Hill and Rice, 1972; Franciosi, 1984; Franciosi and Zaoui, 1991). Many methods have been developed to deal with the issue of this indetermination. For the calculation of slip rates, two major approaches are generally adopted (Busso and Cailletaud, 2005):

- In the elastic-plastic case, the concepts of potentially active $\left(\tau=\tau_{c}\right)$ and effectively active $\left(\tau=\tau_{c}\right.$ and $\left.\dot{\tau}=\dot{\tau}_{c}\right)$ slip systems are introduced. Within the potentially active ones, several subsets of active systems may exist. Thus, it is necessary to find a criterion for selecting the set of effectively active slip systems. Concretely, the combination of slip systems which is chosen as the truly active one is the one that minimizes the deformation energy (Franciosi, 
1984; Franciosi and Zaoui, 1991). From a numerical point of view, this requires doing a combinatory analysis which is known to be excessively time consuming.

- In the viscoplastic case, a nonlinear power law is generally used to relate directly the activity of each slip system to its resolved shear stress value. When using this type of approach, the concept of active slip system is no longer relevant because all slip systems are active but only a few of them have a significant slip rate. Computing time needed for this kind of approach is particularly low.

\subsection{New active slip system detection and slip rate calculation}

In the present work, a new approach to determine the slip system activity in the elastic-plastic context is proposed in order to considerably reduce the computing time (Lorrain et al., 2005). This approach shows advantages similar to those of viscoplastic formulations (easy to use with a reduced computing time), while maintaining the rateindependent nature of the constitutive equations, and leads to the same results as those of elastic-plastic reference models. Note that for the same purpose another approach can be found in the literature, consisting of rounding off the corners of the yield surface, which leads to a regular form of Schmid's law and eliminates the ambiguity in the determination of the active slip systems, without the need of introducing rate-sensitivity (Arminjon 1991; Gambin, 1991, 1992), see also (Peeters et al., 2001b) for an anisotropy based approach. Although the above-mentioned regularization techniques result in a slight deviation from the Schmid law, which can be kept reasonably small by choosing appropriate values for the regularizing parameter, this is largely compensated by their benefits in terms of computational efficiency and straightforward use. While these regularization methods preserve the rate-independent character of the original elastic- 
plastic constitutive equations, some authors proposed viscoplastic-type regularizations. In viscoplasticity, the slip rate on a slip system $g$ is related to the resolved shear stress thanks to a nonlinear function (denoted by $k$ here) as follows:

$$
\dot{\gamma}^{g}=k\left(\tau^{g} / \tau_{c}^{g}\right) \tau^{g}
$$

where the strongly nonlinear function $k\left(\tau^{g} / \tau_{c}^{g}\right)$ compares the resolved shear stress to the reference one. In flow rules like Eq. (9), the slip rate is related to the resolved shear stress in a one-to-one manner, and the issue of choice of active slip systems does not arise. The rate-sensitivity parameter $m$ in power-law relations like $\dot{\gamma}^{g}=\dot{\gamma}_{0} \operatorname{sgn}\left(\tau^{g}\right)\left|\tau^{g} / \tau_{c}^{g}\right|^{m}$ can be regarded as a regularizing parameter, and classical plasticity models are recovered in the limit of large values of $m$ (i.e. when $1 / m$ tends towards zero $(1 / m \rightarrow 0))$. We could have used such viscoplastic-type regularization, but in turn, this would have resulted in a rate-sensitive material model, which is not suitable for strain localization analysis based on Rice's bifurcation approach. Indeed, it is wellknown that no bifurcation (loss of uniqueness of the rate form of constitutive equations) occurs for rate-dependent material models, and material instability for those behavior models is usually tackled by means of alternative approaches including finite element computations (Wu et al., 2007), perturbation methods (Dudzinski and Molinari, 1991) and Marciniak-Kuczynski analyses (Zhou and Neale, 1995).

Unlike regular viscoplastic flow rules, Eq. (8) by itself is not able to provide the slip rate $\dot{\gamma}^{g}$ in an independent and straightforward manner. Instead, this equation involves coupling with other slip systems, due to dislocation interactions, and requires lengthy combinatory analyses, based on energy minimization criteria, for the determination of active slip systems and the corresponding slip rates. To avoid this time consuming 
procedure, and in order to obtain a regularized formulation as advantageous as its viscoplastic counterpart, yet rate-independent, the relation linking slip rate $\dot{\gamma}$ to resolved shear stress rate $\dot{\tau}$ is written as:

$$
\dot{\gamma}^{g}=k^{g}\left(\tau^{g}, \tau_{c}^{g}, \dot{\tau}^{g}\right) \dot{\tau}^{g}
$$

where function $k^{g}$ must fulfill, according to flow rule (8), the following requirements:

- $\quad k^{g}$ must be zero if the resolved shear stress is negative in order to calculate only positive slip rates. Therefore, on each slip system, two senses of slip are defined,

- $\quad k^{g}$ must be zero if the resolved shear stress is inferior to the critical one,

- $\quad k^{g}$ must be zero when unloading occurs even if the resolved shear stress reaches the critical one,

- $\quad k^{g}$ must allow the Schmid law and hardening effects to be correctly described when plastic flow occurs.

Among several possible regularization functions the following form is adopted here:

$$
k^{g}=\frac{1}{H} \frac{1}{2}\left(1+\tanh \left(k_{0} \frac{\tau^{g}}{\tau_{r e f}}\right)\right) \frac{1}{2}\left(1+\tanh \left(k_{1}\left(\frac{\tau^{g}}{\tau_{c}^{g}}-1\right)\right)\right) \frac{1}{2}\left(1+\tanh \left(k_{2} \frac{\dot{\tau}^{g}}{\dot{\tau}_{r e f}}\right)\right)
$$

where $H$ is a hardening parameter, $k_{0}, k_{1}, k_{2}, \tau_{r e f}$ and $\dot{\tau}_{\text {ref }}$ are purely numerical parameters allowing the elastic-plastic transition on a given slip system $g$ to be followed with maximum accuracy (the reference constants $\tau_{\text {ref }}$ and $\dot{\tau}_{\text {ref }}$ only serve to make the parameters $k_{i}$ dimensionless), and ' tanh' stands for the hyperbolic tangent function.

From Eq. (11), and owing to the regularization function properties illustrated in Fig. 1, it can be shown that function $k^{g}$ can satisfy the above-mentioned desired 
requirements, with regard to conditions (8), by selecting properly the numerical parameters:

- The first term of (11), for appropriate regularizing parameters, will be zero if the resolved shear stress is negative and 1 if the resolved shear stress is positive.

- The second term ensures that the resolved shear stress reaches the critical one, i.e. the stress is on the yield surface.

- The third term ensures that the material is under plastic loading. According to Eq. (8), a system is active if the resolved shear stress and its rate reach their critical values, which is equivalent to verifying the plastic loading conditions.

These are only qualitative results, it still has to be verified that the obtained active slip systems are the "right” ones. The values of the purely numerical parameters $k_{0} / \tau_{\text {ref }}, k_{1}$ and $k_{2} / \dot{\tau}_{\text {ref }}$ also need to be given. To this end, a parametric study has been carried out in order to model at best a threshold without having the numerical problems inherent to the used functions. Fig. 1 illustrates the regularizing role of the logistic function, which represents a smooth approximation to the discontinuous Heaviside (unit) step function. In Fig. 2, the results obtained for a loading-unloading-reloading uniaxial tensile test are presented. In this figure, the result is presented for the active slip system that has the largest slip rate. The effect of the smooth approximation introduced by the regularizing functions can be clearly measured since it results in a slight discontinuity of resolved shear stress. Further numerical investigations confirm that this is only due to the numerical approximation that generates some residual slip systems with very small slip rates. Note also that this jump can be gradually reduced by taking 
higher values for parameter $k_{1}$, which corresponds to a sharper transition for the elasticplastic regime.

By taking all the above-mentioned considerations into account, the optimal values for the numerical parameters have been found to be as follows:

- 20 for $k_{1}$ (in order to select only potentially active slip systems), because it is multiplied by small terms when slip system activity holds.

- 1 for $k_{0} / \tau_{\text {ref }}$ and $k_{2} / \dot{\tau}_{\text {ref }}$ because they are multiplied by large quantities when potentially active slip systems are considered.

Note that, in addition to the above mentioned requirements, the choice of values for the regularizing numerical parameters is also dictated by considerations of potential computational difficulties. It is well-known that even for viscoplastic formulations, the difficulty encountered in accurately integrating the constitutive equations is directly related to the value of the exponent of the power-law relation in Eq. (9); the larger the value, the stiffer the equations (Rashid and Nemat-Nasser, 1992). The values of our regularizing parameters have been motivated by a balanced compromise between accuracy and computational efficiency.

\subsection{Hardening}

With the flow rule being specified, it is necessary to determine the hardening law for the single crystal, i.e. the evolution of critical shear stress rate $\dot{\tau}_{c}$ with slip rate $\dot{\gamma}$ for the whole system. This can be done in two ways: 
- The first one relates the rate of the critical shear stress $\dot{\tau}_{c}^{g}$ to the slip rate $\dot{\gamma}^{g}$ by using the hardening matrix that defines self as well as latent hardening (Hill, 1966). The evolution law of the critical shear stress is then given by:

$\dot{\tau}_{c}^{g}=\sum_{h=1}^{n s l i p} H^{g h} \dot{\gamma}^{h}$

nslip is the total number of slip systems and $H^{g h}$ is the hardening matrix.

Phenomenologically, it is difficult to define this matrix to take into account the effect of complex loading paths.

- The second one is based on the description of hardening by dislocation interactions and the link between plastic flow and their motion and storage. The classical relations for a single slip system are (Franciosi, 1984; Tabourot, 1992):

$$
\left\{\begin{array}{l}
\tau_{c}=\tau_{0}+\alpha \mu b \sqrt{\rho} \\
\dot{\rho}=\frac{1}{b}\left(\frac{1}{L}-2 y_{c} \rho\right) \dot{\gamma}
\end{array}\right.
$$

where:

- $\tau_{0}$ is the critical shear stress for a perfect crystal.

- $\quad b$ is the magnitude of the Burgers vector.

- $y_{c}$ is the critical annihilation distance of dislocations, its value is typically some multiples of $b$.

- $L$ is the mean free path of dislocations.

- $\quad \mu$ is the shear modulus.

- $\alpha \approx 0.5$ is a constant related to the stability of dislocation configurations.

- $\rho$ is the dislocation density. 
Relations (13) were extended to multislip case by Franciosi (1984):

$$
\tau_{c}^{g}=\tau_{0}^{g}+\alpha \mu b \sqrt{\sum_{h=1}^{n s l i p} a^{g h} \rho^{h}}
$$

where:

- $\rho^{h}$ is the mean dislocation density for the system $h$.

- $\quad a^{g h}$ is the anisotropy interaction matrix as introduced by Franciosi (1984) and improved by Hoc (1999). Depending on the nature of dislocation interactions (coplanar, collinear systems, etc) the different components are defined by nine parameters. In the case of IF steels, the values of these parameters were identified by Hoc (1999); they will be used for all the steels studied in this work.

By differentiating (14), one obtains the hardening rate for a given slip system as a function of the dislocation density rate of the other systems:

$$
\dot{\tau}_{c}^{g}=\frac{1}{2} \frac{\alpha \mu b}{\sqrt{\sum_{k=1}^{n s l i p} a^{g k} \rho^{k}}} \sum_{h=1}^{n s l i p} a^{g h} \dot{\rho}^{h}
$$

The dislocation density rate $\dot{\rho}^{g}$ of the slip system $g$ was first expressed from slip rate $\dot{\gamma}^{g}$ by Kocks (1976), without specifying the annihilation mechanisms. The recovery term has been derived by Essmann and Mughrabi (1979) in order to take into account the annihilation of close dislocations. The corresponding equation is given by:

$$
\dot{\rho}^{g}=\frac{1}{b}\left(\frac{1}{L^{g}}-2 y_{c} \rho^{g}\right) \dot{\gamma}^{g}
$$

where $L^{g}$ is the mean free path of dislocations on the slip system $g$, given by: 
$\frac{1}{L^{g}}=\frac{1}{D}+\frac{\sqrt{\sum_{h=1, h \neq g}^{n s l i p} \rho^{h}}}{g_{0}}$

- $\quad D$ is the average grain size.

- $g_{0}$ is a parameter related to the dislocation structure (it corresponds to the storage of dislocations).

By combining (16) and (15), the expression of the hardening matrix is obtained:

$$
H^{g h}=\frac{1}{2} \frac{\alpha \mu}{\sqrt{\sum_{k=1}^{n s l i p} a^{g k} \rho^{k}}} a^{g h}\left(\frac{1}{L^{h}}-2 y_{c} \rho^{h}\right)
$$

Expressions (16) and (18) have the same structure: a first term, proportional to the square root of the dislocation densities, corresponding to dislocation storage and a second term, proportional to the dislocation densities, corresponding to their annihilation. This implies that the dislocation densities have a saturation value that depends on material parameters. This is also true for the critical shear stress (Lorrain, 2005) and corresponds to commonly observed phenomena (Peeters et al., 2001a).

\subsection{Qualitative results for the local law}

In order to validate the single crystal model, some results are given in this part:

- Comparison between active slip systems given by this approach and those obtained by classical ones,

- Results in the case of a complex loading path and some effects related to the large strain framework,

- Effect of lattice spin or shear stress rate. 
Finally, softening mechanisms introduced at this scale will be summarized.

\subsubsection{Slip system detection}

In order to demonstrate the validity of the proposed law in detecting slip systems, comparative tests have been simulated and compared with the results from the paper of Nesterova et al. (2001). They simulated shear tests by using the fully-constrained Taylor model and by assuming that slip can occur only on the $<111>\left(\begin{array}{lll}1 & 1 & 0\end{array}\right)$ family. The results of the comparison are reported in Table 2, while the slip system numbering is given in Table 1 . In all of the cases, the major slip systems are correctly predicted, and in only one case a minor slip system is not predicted by the present model. This difference is probably due to the definition of the hardening matrix which is not exactly the same in the two models (it is not given in Nesterova et al. (2001)) and can lead to a slight difference in the selection of minor slip systems. Furthermore, other slip systems are also active in the present model. They are called residual slip systems because their slip rates are at least 100 times smaller than those of the other slip systems. This new law for determining active slip systems is comparable to that of an elastic-viscoplastic model, where all systems are active but only a few of them have significant slip rates (Berbenni, 2002). This validates the method, and thus the new determination of active slip systems can be used much more efficiently than classical approaches based on time consuming combinatory analyses. 


\subsubsection{Effect related to the large strain framework}

Effects introduced by the large strain framework, due to the terms associated with the crystallographic frame rate, are taken into account in the present model. The definition of the resolved shear stress on the slip system $g$ leads to:

$$
\tau^{g}=\sigma: \mathbf{R}^{g}
$$

By differentiating, one obtains:

$$
\dot{\tau}^{g}=\dot{\sigma}: \mathbf{R}^{g}+\sigma: \dot{\mathbf{R}}^{g}
$$

The last expression shows that, if lattice spin $\dot{\mathbf{R}}^{g}$ is sufficiently large, it is possible to have an active slip system while Cauchy's stress is decreasing. Fig. 3 shows the evolution of Cauchy's stress as a function of imposed strain $d_{11}$ during a tensile test for two single crystals with different initial crystallographic orientations. It can be clearly seen that, depending on initial orientation, it is possible to obtain at the first stages of the plastic regime a softening zone immediately followed by a hardening one. In Fig. 4, the evolution of Euler's angles during loading are plotted for the two crystalline orientations. It can be seen that the lattice evolves quickly at the beginning of loading for the crystal that exhibits a softening phase.

\subsection{Remarks and discussion}

In this part, single crystal behavior has been developed and some softening effects have clearly been taken into account and highlighted:

- Multislip activity that more favorably accommodates plastic strain and reduces incompatibilities. 
- Softening terms have been introduced in the hardening matrix by taking into account the annihilation of dislocations.

- Convective terms related to the large strain framework and representing the spin of stressed lattices create softening in some cases at the onset of plastic regime.

The main objective of this work is to take into account the most important softening mechanisms in order to predict loss of ellipticity of the overall behavior with better accuracy. Consequently, the large strain self-consistent scheme in the sense of Hill is presented in the next part to deduce the homogenized macroscopic behavior from the single crystal constitutive law. In the same way as for single crystal model, some results for the polycrystalline behavior are first derived in order to validate the model.

\section{Scale transition scheme}

The aim of a scale-transition scheme is to derive the macroscopic behavior starting from the knowledge of the microscopic one and the microstructure (including texture) of the representative volume element. Since this scheme is now well developed (Iwakuma and Nemat-Nasser, 1984), only the main points of this classical approach are recalled here. The macroscopic behavior law linking the macroscopic nominal stress rate $\dot{\mathbf{N}}$ to the macroscopic velocity gradient $\mathbf{G}$ by the macroscopic tangent modulus $\mathbf{L}$ has the same incremental form as that of the single crystal:

$$
\dot{\mathbf{N}}=\mathbf{L}: \mathbf{G}
$$

The macroscopic nominal stress rate and the velocity gradient are the volume averages of the microscopic ones: 


$$
\left\{\begin{array}{l}
\mathbf{G}=\frac{1}{V} \int_{V} \mathbf{g}(\mathbf{x}) d v=\overline{\mathbf{g}(\mathbf{x})} \\
\dot{\mathbf{N}}=\frac{1}{V} \int_{V} \dot{\mathbf{n}}(\mathbf{x}) d v=\overline{\dot{\mathbf{n}}(\mathbf{x})}
\end{array}\right.
$$

In the self-consistent scale transition scheme, fourth-order concentration tensors, linking macroscopic tensorial fields to their microscopic counterparts, are commonly introduced:

$$
\left\{\begin{array}{l}
\mathbf{g}(\mathbf{x})=\mathbf{A}(\mathbf{x}): \mathbf{G} \\
\dot{\mathbf{n}}(\mathbf{x})=\mathbf{B}(\mathbf{x}): \dot{\mathbf{N}}
\end{array}\right.
$$

By combining Eqs. (1), (21), (22) and (23), the macroscopic tangent modulus is given by:

$$
\mathbf{L}=\frac{1}{V} \int_{V} \mathbf{l}(\mathbf{x}): \mathbf{A}(\mathbf{x}) d v=\overline{\mathbf{l}(\mathbf{x}): \mathbf{A}(\mathbf{x})}
$$

At this stage, it is usually assumed that, for each individual grain, the behavior and the mechanical fields are homogeneous. For a given grain $I$ of volume $V^{I}$, we define the indicator function $\theta^{I}$ by:

$$
\left\{\begin{array}{lll}
\theta^{I}(\mathbf{x})=1 & \text { if } & \mathbf{x} \in V^{I} \\
\theta^{I}(\mathbf{x})=0 & \text { if } & \mathbf{x} \notin V^{I}
\end{array}\right.
$$

this leads to:

$$
\left\{\begin{array}{l}
\mathbf{g}(\mathbf{x})=\sum_{I=1}^{\text {Ngrain }} \mathbf{g}^{I} \theta^{I}(\mathbf{x}) \\
\mathbf{l}(\mathbf{x})=\sum_{I=1}^{\text {Ngrain }} \mathbf{l}^{I} \theta^{I}(\mathbf{x})
\end{array}\right.
$$

where $\mathbf{g}^{I}$ is the volume average of the velocity gradient and $\mathbf{I}^{I}$ is the volume average of the tangent modulus for grain $I$ and Ngrain is the number of grains which make up the polycrystalline aggregate. 
The derivation of the concentration tensor $\mathbf{A}$ that appears in Eqs. (23) and (24) is not a trivial task and requires rather elaborate mathematical development. For this purpose, detailed formulations can be found in the literature, and only the main lines are summarized in what follows, the reader can refer to (Berveiller and Zaoui, 1979; FassiFehri, 1985; Berveiller et al., 1987; Lipinski et al., 1995) for further details. First, field equations (quasi-static equilibrium, compatibility and boundary conditions) are combined making use of Green's tensor techniques to transform the problem into an integral equation; see (Dederichs and Zeller, 1973; Berveiller and Zaoui, 1984), within infinitesimal transformations, and (Lipinski and Berveiller, 1989) at finite deformation. In that process, a reference fictitious homogeneous medium is introduced. Note that within the large strain framework, the Lamé's operator involved in these derivations is not self-adjoint due to the lack of symmetry of the local and overall tangent moduli. Nevertheless, the construction of the integral formulation is still possible using the adjoint operator of the Green tensor as suggested by Willis (1988). From these formal developments, and in order to obtain expressions of practical use, the remaining derivations lie in the appropriate choice for the reference homogeneous medium as well as some simplifications for the integral equation such as the so-called Born approximation. Finally, by decomposing the modified Green tensor into local and nonlocal parts together with some convenient properties (Lipinski and Berveiller, 1989;

Lipinski et al., 1995), the concentration tensor $\mathbf{A}^{I}$ for grain $I$ can be written as:

$$
\mathbf{A}^{I}=\left(\mathbf{I}-\mathbf{T}^{I I}:\left(\mathbf{l}^{I}-\mathbf{L}\right)\right)^{-1}:{\overline{\left(\mathbf{I}-\mathbf{T}^{I I}:\left(\mathbf{l}^{I}-\mathbf{L}\right)\right)^{-1}}}^{-1}
$$

where $\mathbf{T}^{I I}$ is the interaction tensor for grain $I$, related to Eshelby's tensor (Eshelby, 1957) for an ellipsoidal inhomogeneity. Explicit expressions for the interaction tensor 
$\mathbf{T}^{I I}$ can only be found for isotropic media; for general anisotropy such as in the proposed model, an integral over the ellipsoid is obtained with resort to Fourier's transforms (Fassi-Fehri, 1985; Berveiller et al., 1987), and its numerical evaluation is achieved by means of Gauss-Legendre quadrature. In the case of a polycrystalline aggregate comprising Ngrain grains with their respective volume fraction $f^{I}$, the 1 site self-consistent expression corresponding to the self-consistent scheme in the sense of Hill (Hill, 1965a) can be finally obtained as follows:

$$
\mathbf{L}=\sum_{I=1}^{\text {Ngrain }} f^{I} \mathbf{l}^{I}: \mathbf{A}^{I}
$$

The expression of this effective modulus, Eqs. (27) and (28), reveals an implicit

character, since $\mathbf{T}^{I I}, \Delta \mathbf{I}^{I}=\left(\mathbf{l}^{I}-\mathbf{L}\right)$ and thus $\mathbf{A}^{I}$ depend on the yet-unknown modulus L. An iterative procedure is required to conjointly solve Eqs. (27) and (28). In our computational procedure, we adopted an iterative scheme that most often converges within two to five iterations and always within less than ten iterations.

\subsection{Morphological and crystallographic evolution}

It is important to take into account morphological and crystallographic changes for each grain during loading. The change of crystallographic orientation is due to elastic rotation rate $\mathbf{w}^{e}$ as (Nesterova et al., 2001; Iwakuma and Nemat-Nasser, 1984). The relations between the rates of Euler's angles and lattice spin $\mathbf{w}^{e}$ are:

$$
\left\{\begin{array}{l}
\dot{\varphi}_{1}=-w_{12}^{e}+\frac{\cos \phi}{\sin \phi}\left(w_{13}^{e} \cos \varphi_{1}+w_{23}^{e} \sin \varphi_{1}\right) \\
\dot{\phi}=-w_{23}^{e} \cos \varphi_{1}+w_{13}^{e} \sin \varphi_{1} \\
\dot{\varphi}_{2}=-\frac{1}{\sin \phi}\left(w_{13}^{e} \cos \varphi_{1}+w_{23}^{e} \sin \varphi_{1}\right)
\end{array}\right.
$$


where $\varphi_{1}, \phi, \varphi_{2}$ are Euler's angles of a single crystal defining crystallographic orientation according to Bunge’s notation (Bunge, 1969, 1982). A schematic representation defining these three Euler angles is also given in Fig. 5. For the morphology of the grain, the morphological orientation evolves with the total rotation rate as:

$$
\left\{\begin{array}{l}
\dot{\varphi}_{1}^{\prime}=-w_{12}+\frac{\cos \phi^{\prime}}{\sin \phi^{\prime}}\left(w_{13} \cos \varphi_{1}^{\prime}+w_{23} \sin \varphi_{1}^{\prime}\right) \\
\dot{\phi}^{\prime}=-w_{23} \cos \varphi_{1}^{\prime}+w_{13} \sin \varphi_{1}^{\prime} \\
\dot{\varphi}_{2}^{\prime}=-\frac{1}{\sin \phi^{\prime}}\left(w_{13} \cos \varphi_{1}^{\prime}+w_{23} \sin \varphi_{1}^{\prime}\right)
\end{array}\right.
$$

where $\varphi_{1}^{\prime}, \phi^{\prime}, \varphi_{2}^{\prime}$ are Euler's angles of a single crystal defining morphological orientation according to Bunge's notation. These angles define the orientation of the principal coordinate system of the ellipsoid representing the grain relative to the coordinate system (RD, TD, ND) of the sample. The evolution of the shape of the grains taken as ellipsoids is due to the total deformation rate as:

$$
\left\{\begin{array}{l}
\dot{a}=a g_{11}^{\text {ell }} \\
\dot{b}=b g_{22}^{\text {ell }} \\
\dot{c}=c g_{33}^{\text {ell }}
\end{array}\right.
$$

where $a, b$ and $c$ are the half-axes of the ellipsoid representing the shape of the grain, while $\mathbf{g}^{\text {ell }}$ is the projection of the velocity gradient onto the morphological frame.

\subsection{Simulation of the behavior of polycrystalline aggregates}

In this section, the results obtained with the proposed model are compared to experimental ones. Several sequential rheological tests have been performed for two 
steels: a single-phase ferritic steel and a dual-phase (ferrite and martensite) steel. It is necessary to identify the following material parameters for these steels:

- The initial critical shear stress which is assumed to be the same for all slip systems and for each grain of the same phase. In the case of a uniaxial tensile test and for a single phase steel, its value is approximately one half of the elastic limit.

- The parameter $g_{0}$ related to the mean free path of dislocations. Its influence is found to be strong during the first stages of deformation. The global hardening slope is inversely proportional to $g_{0}$.

- The critical annihilation distance $y_{c}$ has a significant effect mainly when deformation becomes large enough. The global hardening slope is inversely proportional to $y_{c}$.

- The average grain size $D$ which can be easily identified using optical micrography.

These parameters are identified by means of two stress-strain curves: a uniaxial tensile test and a Bauschinger shear test. The identified parameters for these two steels are listed in Table 3. It is important to note that the hardening parameters, in the case of the dual-phase steel, have been first taken identically for the two phases, except for the average grain size and the initial critical shear stress, to identify the macroscopic behavior. This choice is made because the strain hardening modes in martensite are not well-known and this reduces the number of parameters that need to be identified.

Moreover, much attention has also been paid to numerical procedures: the singlecrystal behavior law is implemented using the fourth-order Runge-Kutta algorithm, 
more robust and reliable than forward Euler scheme; the strongly implicit selfconsistent problem is solved using a classical convergence algorithm. A method to invert ill-conditioned matrices encountered during loading is also used in order to simulate the loading path with better accuracy (this method uses the inverse of the previous calculation step to compute the inverse in the current step).

Note also that this choice of explicit scheme has been motivated by a compromise with regard to considerations of efficiency, accuracy and convergence. Indeed, phenomenological behavior models are governed by constitutive equations that have similar forms (a set of ordinary differential equations and some algebraic equations), and numerical integration procedures for those models have been precisely described in the literature (Hughes, 1984; Simo and Taylor, 1985; Ortiz and Popov, 1985). The respective benefits and drawbacks with regard to accuracy, stability, robustness and efficiency of various implicit and explicit time integration schemes have been investigated and discussed by many authors. However, for constitutive equations with a large number of internal variables, implicit integration procedures are often avoided and instead, explicit schemes are adopted. The main reason for this is that when behavior models with a large number of internal variables are considered, implicit schemes lead to a large size linear algebraic system to be solved by means of successive iterations, and involve inverting large size matrices, which can substantially affect efficiency and even convergence. A former study on the time integration of advanced elastic-plastic constitutive models having a large number of internal variables, yet phenomenological (Haddag et al., 2007), has confirmed that adequate alternatives would be either to reduce the size of the nonlinear system to be solved (whenever it is possible), or to adopt an explicit time integration scheme. In addition to the explicit fourth-order 
Runge-Kutta algorithm used for the integration of the proposed finite-deformation selfconsistent model, and in order to preserve both accuracy and stability, the loading increments are kept small enough and an adaptive time step is used in order to avoid loss of accuracy when changing loading paths are considered (especially for the transition from elastic to plastic regime).

In Figs. 6 and 7 comparisons between simulations and experiments for these two steels are plotted. Only the uniaxial tension and the Bauschinger shear test until $10 \%$ of logarithmic strain for the first path have been used to identify the material parameters, the other results have been used for validation purposes. As observed in Fig. 6, numerical results are in good agreement with the experimental ones. However, some gaps appear especially at the onset of the plastic regime during the second loading path for Bauschinger tests and orthogonal test. These gaps increase as pre-strain gets larger. They can be explained by the fact that the spatial rearrangement of dislocation cells is not taken into account in the present model (Lorrain, 2005). Experimental evidence of such a rearrangement can be found easily in the literature for IF-steels (Nesterova et al., 2001). In the present model, the internal variable acting at this scale is the mean dislocation density for a slip system. At the onset of the second loading path, the structure of the dislocation cells is disintegrated and another one depending on the second loading path is created (Peeters, 2002), which may explain the observed differences. In order to reduce this gap an idea would consist of introducing kinematic hardening at the slip system level (the kinematic hardening in the present model is only inter-granular and not intra-granular due to the self-consistent scheme). By introducing this variable, the proposed model should be able to accurately account for the effect of dislocation cells (Berbenni, 2002). The same remarks apply to Fig. 7. The agreement in 
this case is better due to the less-pronounced effect of dislocation cells for this material in comparison to the first one. On the whole, these two figures show that the proposed model is able to reproduce the elastic-plastic behavior of single and dual phase polycrystalline materials.

This model has been validated for several other steels and allows the simulation of Lankford's coefficients, yield surfaces and texture evolution with good correlation when compared to experimental results (Lorrain, 2005).

In the next part, Rice's criterion, which is based on the loss of ellipticity of the partial differential equations governing the boundary value problem, is introduced and combined with the micro-macro constitutive law presented and validated in the two previous parts. The results obtained with this criterion for different steels are used to plot direct and sequential Ellipticity Limit Diagrams (ELDs).

\section{Ductility loss modeling}

\subsection{Strain localization criterion}

The so-called Rudnicki-Rice criterion (Rudnicki and Rice, 1975; Rice, 1976; Asaro, 1983; Peirce, 1983; Peirce et al., 1983; Petryk, 2000) corresponds to a bifurcation associated with admissible jumps for strain and stress rates across a shear band as illustrated in Fig. 8.

Since field equations have to be satisfied, the kinematic condition for the strain rate jump is:

$$
[\mathbf{G}]=\mathbf{G}^{+}-\mathbf{G}^{-}=\kappa \otimes v
$$

where $\kappa$ is the jump amplitude and $v$ is the unit normal to the shear band. 
On the other hand, the continuity of the stress rate vector has to be verified for the forces along the interface created by the localization band:

$$
\dot{\mathbf{N}}^{T+} \cdot \mathrm{v}=\dot{\mathbf{N}}^{T-} \cdot \mathrm{v} \Rightarrow\left[\dot{\mathbf{N}}^{\mathrm{T}}\right] \cdot \mathrm{v}=\mathbf{0}
$$

By using the constitutive law (21), one has:

$$
\left(\mathbf{L}^{+}: \mathbf{G}^{+}\right)^{T} \cdot \mathbf{v}=\left(\mathbf{L}^{-}: \mathbf{G}^{-}\right)^{T} \cdot \mathbf{v}
$$

This can be written in the form:

$$
\left(\mathbf{L}^{+}:(\kappa \otimes \mathbf{U})\right)^{T} \cdot \mathbf{U}=\left(\left(\mathbf{L}^{-}-\mathbf{L}^{+}\right): \mathbf{G}^{-}\right)^{T} \cdot \mathbf{U}
$$

At the onset of the bifurcation, it can be considered that the tangent modulus is the same on each part of the band:

$$
\mathbf{L}^{+}=\mathbf{L}^{-}=\mathbf{L}
$$

Therefore (35) can be put in the form:

$$
(\mathbf{L}:(\kappa \otimes \mathbf{u}))^{T} \cdot \mathbf{u}=\mathbf{0}
$$

which is equivalent to:

$$
(v \cdot \mathbf{L} \cdot \mathbf{u}) \cdot \kappa=\mathbf{0}
$$

From Eq. (38), two families of solutions can be extracted. The first possibility is when all of the $\kappa \mathrm{s}$ are zero and thus the magnitude of the jumps is zero, in which case there is no bifurcation. The second possibility corresponds to the existence of at least one non zero $\kappa$, in which case the system of Eqs. (38) has an infinite number of solutions leading to the following condition:

$$
\operatorname{det}(\mathbf{v} \cdot \mathbf{L} \cdot \mathbf{u})=0
$$

Each direction of the space verifying (39) corresponds to a localization band where the amplitude of the jump cannot be calculated directly. 


\section{ACCEPTED MANUSCRIPT}

Eq. (39) is the so-called Rudnicki-Rice criterion corresponding to the loss of ellipticity of the boundary value problem. Some contributions (Bigoni and Hueckel, 1991; Keryvin, 1999) have also been made using the strong ellipticity loss criterion and Hill’s criterion (Hill, 1958). They have demonstrated that for associated plasticity models and for small strain framework, the ellipticity loss and strong ellipticity loss criteria are equivalent.

It has to be noted that no additional parameters have been employed here, this criterion being intrinsic. This is a strong advantage over the M-K classical approach. Recently, some enhanced models with an evolving internal length scale have been introduced to capture strain localization related to shear band thickness (Voyiadjis et al., 2005; Vrech and Etse, 2006).

In (39), ductility loss is reached when the first-zero eigenvalue is seen in the acoustic tensor, but in the case of a polycrystalline aggregate where ductility loss is due to a collective effect, some work must be done before applying this criterion. If an individual grain reaches this criterion, this is not necessarily the case also for the polycrystal. To highlight this problem, a tensile test on a 1000-grain polycrystalline aggregate is simulated and the criterion is applied for each grain as well as for the whole polycrystal. The volume fraction of grains that have reached their ductility limit (the criterion (39) is applied for each tangent modulus $\mathbf{l}$ of each grain) and the minimum

value of $(\operatorname{det}(v \cdot \mathbf{L} \cdot \boldsymbol{v}))^{1 / 3}$ for all of the orientations $\boldsymbol{v}$, where $\mathbf{L}$ is the tangent modulus for the polycrystal, are plotted together in Fig. 9 as functions of the macroscopic strain. It appears that the loss of ellipticity for the polycrystalline tangent modulus occurs when nearly all the grains of the polycrystal individually satisfy this criterion. This result implies that: 
- This criterion has to be applied to the polycrystalline tangent modulus.

- No additional parameter such as a critical volume fraction for macroscopic localization needs to be introduced.

Since forming limits at localization are commonly obtained by using other approaches which are more popular in the literature dealing with sheet metal forming processes, it appears necessary to clearly distinguish between the two concepts: loss of ellipticity and limit of forming. Consequently, we suggest the introduction of the Ellipticity Limit Diagrams (ELDs) corresponding to principal strain values leading to loss of ellipticity during a given loading path, in addition to the classical Forming Limit Diagrams (FLDs).

\subsection{Prediction of Ellipticity Limit Diagrams}

As stated in the introduction, forming limit strains in the sheet metal forming industry is a useful tool for quantifying metals in terms of ductility. However, the experimental measurement of these limit strains is a difficult, time consuming and expensive process. Therefore, it would be very convenient if a predictive, combined theoretical-numerical model could replace many of the experimental FLD measurements. In this part, the ellipticity loss analysis is applied to the two steels studied in the previous part, allowing ELDs to be simulated and then compared to the FLDs provided by ArcelorMittal. The FLDs from ArcelorMittal were obtained using a model developed by Cayssials (Cayssials, 1998; Cayssials and Lemoine, 2005). This model, commonly used by ArcelorMittal, has proven to be reliable in predicting formability for linear loading paths. In particular, the validity of this ArcelorMittal’s FLD model has been assessed through a wide range of grades of sheet metals, for which 
experimental FLDs have been simultaneously measured. For linear loading paths, the results of this model are shown to be in agreement with experiments (cf. Fig. 10). Despite its good results, its restriction to linear loading paths together with its phenomenological basis, in which microstructural effects cannot be accounted for, have motivated the development of the proposed approach with the aim of designing new materials. The results given by the proposed model based on Rice’s localization criterion are reported in Figs. 11 and 12 and compared to ArcelorMittal's FLDs and experiments. Note that only one experimental point, corresponding to the plane tensile test, is available for the two steels under consideration. As expected, some differences in position may appear between ELDs and FLDs, mainly due to:

- In the case of simulation, localization is reached when Rice's criterion is verified, i.e. when deformation no longer remains "macro-homogeneous" in the specimen. This definition may sometimes be restrictive. Aside from this, the micro-macro modeling does not introduce a structural effect but only a material effect. This modeling corresponds to one material point of the specimen.

- Experimentally, the FLD represents localized necking for different loading paths (uniaxial tensile test, biaxial tests, plane strain test). To obtain these different paths, Marciniak's or Nakazima's punch of 100 mm diameter with specimens of different widths is used. From a test until failure, the measure of strains is achieved from a $2 \mathrm{~mm}$ square grid on a $40 \times 10 \mathrm{~mm}^{2}$ zone centered on the observed crack. After the determination of the necking points from the second derivative method (gradient of deformation), the FLD point is obtained by interpolation of the strain profile across the crack (where only the points that do not exhibit necking are kept) and by finding the maximum (generally positioned 
on the crack). The FLD is plotted using the necking points for different paths. This method of analysis introduces a structural effect. That is to say that when the size of specimen is changed, the size of square grid must be changed ( $1 \mathrm{x} 1$ $\mathrm{mm}^{2}$ instead of 2x2 $\mathrm{mm}^{2}$ ) to obtain the same necking point (Geoffroy and Adami, 2005).

As it can also be seen in Figs. 11 and 12, some localization points, given by the model, exhibit a certain dispersion in the expansion domain, especially for the single phase steel (Fig. 11). It is mainly due to statistics related to grain to grain heterogeneities. Further investigations are currently being done to better understand this phenomenon. In order to obtain a representative curve allowing comparison with FLDs, a polynomial interpolation has been used to plot the ELDs. In spite of the observed difference due to the above-mentioned structural effect, the ELDs (cf. Figs. 11 and 12) not only are close to the reference FLDs provided by ArcelorMittal, but also allow reproducing the impact of metallurgy on the formability when designing new grades of steel. In Figs. 13 and 14, ELDs are plotted with two different pre-strain for the two steels. Only a qualitative comparison can be made because no experimental or reference results were available, as ArcelorMittal's FLD model is not designed to predict formability when two-stage or more complex loading paths are considered. Haddad (1997) has shown that during a second loading path, the FLD is shifted in the direction of the first path. For example, in the case of uniaxial tension pre-strain the curve must be translated along the preloading direction. This is clearly observed in Figs. 13 and 14.

It is also interesting to investigate the impact of microstructural mechanisms on ductility, which can be advantageously used in the design of new materials in order to optimize their formability and mechanical properties in-use. The effect of four 
parameters has been analyzed, namely (the average grain size $D$, the critical annihilation distance of dislocations $y_{c}$, the parameter $g_{0}$ related to storage of dislocations and the initial critical shear stress $\tau_{0}^{g}$ ). The results obtained relative to these effects correspond to common experimental observations; for example, reducing the average grain size produces higher-strength materials but, in turn, induces a drop in ductility. The impact of softening due to dislocation annihilation is chosen in particular, to be emphasized in the following paragraph.

Locking again at Eq. (16), which gives the evolution of dislocations, reveals that it contains two terms of opposite sign corresponding respectively to storage and annihilation of dislocations. The importance of annihilation can be quantified by means of a material parameter $y_{c}$, corresponding to the critical annihilation distance of dislocations, the larger this parameter, the more dislocations are annihilated. The mathematical structure of the ordinary differential equation (16) suggests that it can be integrated in the form of a saturating response, as long as annihilation is present in the model. In the limit of a vanishing parameter $y_{c}$, no saturation is reached and, in addition, this parameter is shown to control the saturation rate: larger values of this parameter lead to a quicker saturation. Therefore, this softening effect, due to dislocation annihilation, contributes to the overall softening of the material thus promoting strain localization instabilities.

To be more specific, this impact of softening, due to dislocation annihilation, on ductility is quantified using a uniaxial tensile test for a single-phase steel together with Rice’s bifurcation criterion (cf. Fig. 15). By considering various annihilation states, corresponding to different values of parameter $y_{c}$, it is clearly shown (Fig. 15) that 
larger values of $y_{c}$ induce more annihilation and the corresponding softening effect is a limiting factor for ductility since it promotes early strain localization.

In the same way, the three-dimensional effect of necking analysis will be emphasized in this remaining section of the paper. Indeed, as mentioned in the introduction, our approach based on Rice's bifurcation criterion allows a fully three-dimensional analysis of shear band formation. In contrast to most of the previously published contributions, based on plane-stress assumptions, the proposed model is able to give information regarding the through-thickness orientation of the localized band. As shown in Fig. 16, the band orientation can be defined thanks to two angles: the inclination of the band $\theta_{1}$ with respect to rolling direction RD in the sheet plane (RD, TD), and its inclination $\theta_{2}$ with respect to rolling direction RD in the thickness plane (RD, ND). Two different loadings - uniaxial tension and plane tension - for the two steels have been considered and the results of orientation analysis are reported in Table 4. For the uniaxial tensile test, the in-plane band inclination with respect to rolling direction is found to be $52^{\circ}$ for the single-phase steel and $51^{\circ}$ for the dual-phase steel. These results are close to the value of $54^{\circ}$ generally observed in experiments. This latter in-plane orientation can also be recovered by means of Hill’s criterion (Hill, 1950), which gives a direct relation between the angle and the principal strain ratio. Doghri and Billardon (1995) also found this value for the in-plane orientation using both an analytical formulation and an elastic-plastic model coupled with damage implemented into a finite element code. Another recent contribution (Haddag et al., 2006, 2008), in which Rice’s bifurcation criterion has been used in conjunction with an advanced elastic-plastic model coupled with damage, also provides the value of $54^{\circ}$ for the in-plane orientation. Detailed investigations in this regard (Kuroda and Tvergaard, 2007) reveal that the orientation of 
shear bands can depend on the texture and anisotropy. Texture effects may explain the slight difference between the above-mentioned results. With regard to thickness, the proposed model predicts a band perpendicular to the sheet plane for both single-phase as well as dual-phase steel, which is in agreement with the investigations of Haddag et al. (2006, 2008).

For the plane tensile test, the model predicts a band perpendicular to the rolling direction for the two steels (i.e. the in-plane orientation with respect to rolling direction is $\theta_{1}=90^{\circ}$ ), which is in agreement with many experiments and also with recently published contributions (Kuroda and Tvergaard, 2007; Haddag et al., 2006, 2008). For the out-of-plane inclination of the band, the model gives the same value for the two steels: $46^{\circ}$ with respect to rolling direction; once again these results are in agreement with previous analyses (Haddag et al., 2006, 2008).

\section{Conclusion}

In this paper an advanced micro-macro approach has been combined with Rice’s criterion in order to plot explicit ELDs and compare them to experimental and reference FLDs. For this purpose, the macroscopic behavior law has been accurately defined in order to take into account the most important softening mechanisms so that the effects of complex loading paths are (at least partially) captured. The introduced ductility criterion allows the determination of ELDs which have a very similar form to experimental FLDs and reproduce qualitatively the effects of complex loading paths. This theoretical and numerical tool allows predicting the ductility of new materials since the early stage of the design of new grades of steel and thus provides a powerful and useful tool for steelmakers like ArcelorMittal. Its major interest lies in its capability 
of comparing the formability of these materials by assessing the impact of microstructural effects on ductility. Therefore, it can be advantageously used to optimize the ductility of new steels or to design materials with desired formability. In contrast to more phenomenological approaches, it is worthwhile to notice that the proposed model does not need any additional parameter to capture the occurrence of strain localization.

\section{Acknowledgements}

This research has been performed within a project funded by ArcelorMittal Research Group. The authors are grateful to ArcelorMittal Research that provided them with FLDs experimental data allowing interesting comparisons. The authors would also like to thank ArcelorMittal Research for its financial support. 


\section{References}

Aretz, H., 2007. Numerical analysis of diffuse and localized necking in orthotropic sheet metals. Int. J. Plasticity 23, 798-840.

Aretz, H., 2008. A simple isotropic-distortional hardening model and its application in elastic-plastic analysis of localized necking in orthotropic sheet metals. Int. J. Plasticity, doi:10.1016/j.ijplas.2007.10.002.

Arminjon, M., 1991. A regular form of the Schmid law. Application to the ambiguity problem. Textures and Microstructures 14-18, 1121-1128.

Arrieux, R., 1995. Determination and use of the forming limit stress diagrams in sheet metal forming. J. Mat. Proc. Tech. 53, 47-56.

Asaro, R.J., 1983. Crystal plasticity. J. App. Mech. 50, 921-934.

Asaro, R.J., Rice, J.R., 1977. Strain localization in ductile single crystals. Journal of the Mechanics and Physics of Solids 25, 309-338.

Barata Da Rocha, A., 1989. Theoretical forming limit diagrams of anisotropic sheets Linear and non-linear strain paths. In: Wagoner, R.H., Chan, K.S., Keeler, S.P. (Eds.), Forming Limit Diagrams: Concepts Methods and Applications, pp. 183-201.

Barlat, F., 1989. Forming limit diagrams - Predictions based on some microstructural aspects of materials. In: Wagoner, R.H., Chan, K.S., Keeler, S.P. (Eds.), Forming Limit Diagrams: Concepts Methods and Applications, pp. 275-301. 
Baudelet, B., 1984. Prediction of forming limit diagrams for deep drawing. Agard Lecture Series 137 (3), 1-22.

Berbenni, S., 2002. Elastoviscoplasticité des matériaux polycristallins: Modélisation micromécanique et physique. Application au comportement dynamique et à l'effet Bake-Hardening. PhD Thesis ENSAM Metz, France.

Berveiller, M., Fassi-Fehri, O., Hihi, A., 1987. The problem of two plastic and heterogeneous inclusions in an anisotropic medium. Int. J. Eng. Sci. 25(6), 691-709.

Berveiller, M., Zaoui, A., 1979. An extension of the self-consistent scheme to plastically-flowing polycrystals. Journal of the Mechanics and Physics of Solids 26, 325-344.

Berveiller, M., Zaoui, A., 1984. Modeling of the plastic behaviour of inhomogeneous media. J. Eng. Mat. Tech. 106, 295-299.

Bigoni, D., Hueckel, T., 1991. Uniqueness and localization - I Associative and nonassociative elastoplasticity. Int. J. Solids Struct. 28, 197-213.

Borg, U., 2007. Strain gradient crystal plasticity effects on flow localization, Int. J. Plasticity 23, 1400-1416.

Boudeau, N., Gelin, J.C., Salhi, S., 1998. Computational prediction of the localized necking in sheet forming based on microstructural material aspects. Comp. Mat. Sci. 11, 45-64.

Bunge, H.J., 1969. Mathematische Methoden der Texturanalyse. Akademie-Verlag, Berlin. 


\section{ACCEPTED MANUSCRIPT}

Bunge, H.J., 1982. Texture Analysis in Materials Science. Butterworths, London.

Busso, E.P., Cailletaud, G., 2005. On the selection of active slip systems in crystal plasticity. Int. J. Plasticity 21, 2212-2231.

Butuc, M.C., Gracio, J.J., Barata da Rocha, A., 2006. An experimental and theoretical analysis on the application of stress-based forming limit criterion. Int. J. Mech. Sci. 48, 414-429.

Cayssials, F., 1998. A new method for predicting FLC. In: $20^{\text {th }}$ IDDRG Conference. Brussels.

Cayssials, F., Lemoine, X., 2005. Predictive model of FLC (Arcelor model) upgraded to UHSS Steels. In: $24^{\text {th }}$ IDDRG Conference. Besançon.

Chow, C.L., Jie, M., 2004. Forming limits of Al 6022 sheets with material damage consideration-theory and experimental validation. Int. J. Mech. Sci. 46, 99-122.

Chow, C.L., Yu, L.G., Tai, W.H. Demeri, M.Y., 2001. Predictions of forming limit diagrams for Al6111-T4 under non-proportional loading. Int. J. Mech. Sci. 43, 471-486.

Csontos, A.A., Starke, E.A., 2005. The effect of inhomogeneous plastic deformation on the ductility and fracture behavior of age hardenable aluminium alloys. Int. J. Plasticity 21, 1097-1118.

Dederichs, P.H., Zeller, R., 1973. Variational treatment of the elastic constants of disordered materials. Zeitschrift für Physik A Hadrons and Nuclei 259, 103-116. 


\section{ACCEPTED MANUSCRIPT}

Doghri, I., Billardon, R., 1995. Investigation of localization due to damage in elastoplastic materials. Mechanics of Materials 19, 129-149.

Dudzinski, D., Molinari, A., 1991. Perturbation analysis of thermoviscoplastic instabilities in biaxial loading. Int. J. Solids Struct. 27 (5), 601-628.

Eshelby, J., 1957. The determination of the elastic field of an ellipsoidal inclusion and related problems. Proc. R. Soc. Lond. A 241, 376-396.

Essmann, U., Mughrabi, H., 1979. Annihilation of dislocations during tensile tests and cyclic deformation and limit of dislocations densities. Phil. Mag. A. 40 (6), 731-756.

Fassi-Fehri, O., 1985. Le problème de la paire d'inclusions plastiques et hétérogènes dans une matrice anisotrope - Application à l'étude du comportement des matériaux composites et de la plasticité. PhD thesis, Metz University, France.

Franciosi, P., 1984. Etude théorique et expérimentale du comportement élastoplastique de monocristaux métalliques se déformant par glissement : Modélisation pour un chargement complexe quasi-statique. PhD thesis, Paris North University, France.

Franciosi, P., Zaoui, A., 1991. Crystal hardening and the issue of uniqueness. Int. J. Plasticity 7, 295-311.

Gambin, W., 1991. Crystal plasticity based on yield surfaces with rounded-off corners. Zeitschrift für Angewandte Mathematik und Mechanics, 71(4), T265.

Gambin, W., 1992. Refined analysis of elastic-plastic crystals. Int. J. Solids Struct. 29(16), 2013-2021. 
Geoffroy, J.L., Adami, J.P., 2005. Influence of the specimen geometry for the determination of the Uniaxial Tension necking point for FLD. In: $24^{\text {th }}$ IDDRG Conference. Besançon.

Goya, M., Ito, K., 1990. An expression of elastic-plastic constitutive law incorporating stress increment dependence (initial material with Mises type plastic potential). JSME Int. J. Series 1. 33 (1), 57.

Graf, A., Hosford, W.F., 1983. Calculations of forming limit diagrams for changing strain paths. Met. Trans. 24A, 2497-2501.

Haddad, A., 1997. Contribution à la détermination des courbes limites de formage en contrainte et en déformation à partir de la théorie 3G. PhD Thesis, Savoie University, France.

Haddag, B., Abed-Meraim, F., Balan, T., 2006. Strain localization analysis using large deformation anisotropic elastoplastic model coupled with damage. In: $3^{\text {rd }}$ ECCM Conference, Lisbon.

Haddag, B., Balan, T., Abed-Meraim, F., 2007. Investigation of advanced strain-path dependent material models for sheet metal forming simulations. Int. J. Plasticity 23 (6), $951-979$.

Haddag, B., Abed-Meraim, F., Balan, T., 2008. Strain localization analysis using large deformation anisotropic elastic-plastic model coupled with damage. Submitted to European Journal of Mechanics - A/Solids. 
Harewood, F.J., McHugh, P.E., 2006. Investigation of finite element mesh independence in rate dependent materials. Comp. Mater. Sci. 37, 442-453.

Hill, R., 1950. The Mathematical Theory of Plasticity. Oxford University Press, Oxford.

Hill, R., 1952. On discontinuous plastic states, with special reference to localized necking in thin sheets. Journal of the Mechanics and Physics of Solids 1, 19-30.

Hill, R., 1958. A general theory of uniqueness and stability in elastic-plastic solids. Journal of the Mechanics and Physics of Solids 6, 239-249.

Hill, R., 1965a. Continuum micro-mechanics of elastoplastic polycrystals. Journal of the Mechanics and Physics of Solids 13, 89-101.

Hill, R., 1965b. A self consistent mechanics of composite materials. Journal of the Mechanics and Physics of Solids 13, 213-221.

Hill, R., 1966. Generalized constitutive relations for incremental deformation of metal crystals by multislip. Journal of the Mechanics and Physics of Solids 14, 95-102.

Hill, R., Rice, J.R., 1972. Constitutive analysis of elastic-plastic crystals at arbitrary strain. Journal of the Mechanics and Physics of Solids 20, 401-413.

Hiwatashi, S., Van Bael, A., Van Houtte, P., Teodosiu, C., 1998. Predictions of forming limit strains under strain-path changes: applications of an anisotropic model based on texture and dislocation structure. Int. J. Plasticity 14, 647-669.

Hoc, T., 1999. Etudes expérimentales et numériques de la localisation de la déformation dans les aciers doux. PhD Thesis, Ecole Centrale de Paris, France. 
Hoc, T., Rey, C., Raphanel, J.L., 2001. Experimental and numerical analysis of localization during sequential test for an IF-Ti steel. Acta Mater. 49, 1835-1846.

Horstemeyer, M.F., 2000. A numerical parametric investigation of localization and forming limits. Int. J. Damage Mech. 9, 255-285.

Hughes, T.J.R., 1984. Numerical implementation of constitutive models: rateindependent deviatoric plasticity. In: Theoretical Foundation for Large-scale Computations for Nonlinear Material Behavior. Martinus Nij Publishers, Netherlands, pp. 29-57.

Hutchinson, J.W., Neale, K.W., 1978. Sheet necking - II. Time-independent behavior. In: Koistinen, D.P., Wang, N.M. (Eds.), Mechanics of Sheet Metal Forming. Plenum Press, New York, pp. 127-153.

Inal, K., Neale, K.W., Aboutajeddine, A., 2005. Forming limit comparisons for FCC and BCC sheets. Int. J. Plasticity 21, 1255-1266.

Ito, K., Satoh, K., Goya, M., Yoshida, T., 2000. Prediction of limit strain in sheet metal forming processes by 3D analysis of localized necking. Int. J. Mech. Sci. 42, 22332248.

Iwakuma, T., Nemat-Nasser, S., 1984. Finite elastic-plastic deformation of polycrystalline metals and composite. Proc. R. Soc. Lond. A 394, 87-119.

Keryvin, V., 1999. Contribution à la modélisation de l'endommagement localisé. PhD Thesis, Poitiers University, France. 
Knockaert, R., Chastel, Y., Massoni, E., 2002. Forming limits prediction using rateindependent polycrystalline plasticity. Int. J. Plasticity 18, 231-247.

Kocks, U.F., 1976. Laws for work-hardening and low-temperature creep. J. Eng. Mat. Tech. 98, 76-85.

Kuroda, M., Tvergaard, V., 2000. Forming limit diagrams for anisotropic metal sheets with different yield criteria. Int. J. Solids Struct. 37, 5037-5059.

Kuroda, M., Tvergaard, V., 2007. Effects of texture on shear band formation in plane strain tension/compression and bending. Int. J. Plasticity 23, 244-272.

Kuwabara, T., 2007. Advances in experiments on metal sheets and tubes in support of constitutive modeling and forming simulations. Int. J. Plasticity 23, 385-419.

Lipinski, P., Berveiller, M., 1989. Elastoplasticity of micro-inhomogeneous metals at large strains. Int. J. Plasticity 5, 149-172.

Lipinski, P., Berveiller, M., Reubrez, E., Morreale, J., 1995. Transition theories of elastic-plastic deformation of metallic polycrystals. Arch. Appl. Mech. 65, 291-311.

Lorrain, J.P., 2005. Critère de ductilité basé sur la perte d'ellipticité du module tangent élastoplastique déduit d'un modèle autocohérent. PhD Thesis, ENSAM Metz, France.

Lorrain, J.P., Ben Zineb, T., Abed-Meraim, F., Berveiller, M., 2005. Ductility Loss Modelling for BCC Single Crystals. Int. J. Forming Processes 8 (2-3), 135-158.

Marciniak, Z., Kuczynski, K., 1967. Limit Strains in processes of stretch-forming sheet metal. Int. J. Mech. Sci. 9 (9), 609-620. 
Menzel, A., Ekh, M., Runesson, K., Steinmann, P., 2005. A framework for multiplicative elastoplasticity with kinematic hardening coupled to anisotropic damage. Int. J. Plasticity 21, 397-434.

Nesterova, E.V., Bacroix, B., Teodosiu, C., 2001. Microstructure and texture evolution under strain path changes in low-carbon interstitial-free steel. Met. Mat. Trans. A 32A, $2527-2538$.

Ortiz, M., Popov, E.P., 1985. Accuracy and stability of integration algorithms for elastoplastic constitutive relations. Int. J. Numer. Methods Engng. 21, 1561-1576.

Peeters, B., 2002. Multiscale modelling of the induced plastic anisotropy in IF steel during sheet forming. PhD Thesis, Katholieke Universiteit Leuven, Belgium.

Peeters, B., Bacroix, B., Teodosiu, C., Van Houtte, P., Aernoudt, E., 2001a. WorkHardening/softening behavior of B.C.C. polycrystals during changing strain path: Part II. TEM observations of dislocation sheets in an IF steel during two stage strain paths and their representation in terms of dislocation densities. Acta Mater. 49, 1621-1632.

Peeters, B., Seefeldt, M., Van Houtte, P., Aernoudt, E., 2001b. Taylor ambiguity in BCC polycrystals: a non-problem if substructural anisotropy is considered. Scripta Mater. 45, 1349-1356.

Peirce, D., 1983. Shear band bifurcations in ductile single crystals. Journal of the Mechanics and Physics of Solids 31 (2), 133-153.

Peirce, D., Asaro, R.J., Needleman, A., 1982. An analysis of nonuniform and localized deformation in ductile single crystals. Acta metal. 30, 1087-1119. 
Peirce, D., Asaro, R.J., Needleman, A., 1983. Material rate dependence and localized deformation in crystalline solids. Acta metal. 31 (12), 1951-1976.

Petryk, H., 2000. General condition for uniqueness in materials with multiple mechanism of inelastic deformation. Journal of the Mechanics and Physics of Solids 48, 367-396.

Rashid, M.M., Nemat-Nasser, S., 1992. A constitutive algorithm for rate-dependent crystal plasticity. Comp. Methods Appl. Mech. Engng. 94, 201-228.

Rice, J.R., 1976. The localization of plastic deformation. In: $14^{\text {th }}$ International Congress of Theoretical and Applied Mechanics, pp. 207-220.

Rudnicki, J.W., Rice, J.R., 1975. Conditions for the localization of deformation in pressure-sensitive dilatant materials. Journal of the Mechanics and Physics of Solids 23, 371-394.

Sánchez, P.J., Huespe, A.E., Oliver, J., 2008. On some topics for the numerical simulation of ductile fracture. Int. J. Plasticity, doi:10.1016/j.ijplas.2007.08.004.

Signorelli, J.W., Bertinetti, M.A., Turner, P.A., 2008. Predictions of forming limit diagrams using a rate-dependent polycrystal self-consistent plasticity model. Int. J. Plasticity, doi:10.1016/j.ijplas.2008.01.005.

Simha, C.H.M., Grantab, R., Worswick, M.J., 2007. Computational Analysis of StressBased Forming Limit Curves. Int. J. Solids Struct. 44, 8663-8684.

Simo, J., Taylor, R., 1985. Consistent tangent operators for rate-independent elastoplasticity. Comput. Methods Appl. Mech. Engng. 48, 101-118. 
Stoughton, T.B., 2000. A general forming limit criterion for sheet metal forming. Int. J. Mech. Sci. 42, 1-27.

Stoughton, T.B., Yoon, J.W., 2005. Sheet metal formability analysis for anisotropic materials under non-proportional loading. Int. J. Mech. Sci. 47, 1972-2002.

Stoughton, T.B., Zhu, X., 2004. Review of theoretical models of the strain-based FLD and their relevance to the stress-based FLD. Int. J. Plasticity 20, 1463-1486.

Tabourot, L., 1992. Loi de comportement élastoviscoplastique du monocristal en grandes déformations. PhD Thesis, Grenoble University, France.

Tang, C.Y., Tai, W.H., 2000. Material damage and forming limits of textured sheet metals. J. Mat. Proc. Tech. 99, 135-140.

Vacher, P., Arrieux, R., Tabourot, L., 1998. Analysis of a criterion of deep drawing operation capability for thin orthotropic sheets. J. Mat. Proc. Tech. 78, 190-197.

Varma, N.S.P., Narasimhan, R., Luo, A.A., Sachdev, A.K., 2007. An analysis of localized necking in aluminium alloy tubes during hydroforming using a continuum damage model. Int. J. Mech. Sci. 49, 200-209.

Viatkina, E.M., Brekelmans, W.A.M., Geers, M.G.D., 2005. A crystal plasticity based estimate for forming limit diagrams from textural inhomogeneities. J. Mat. Proc. Tech. 168, 211-218.

Voyiadjis, G.Z., Alsaleh, M.I., Alshibli, K.A., 2005. Evolving internal length scales in plastic strain localization for granular materials. Int. J. Plasticity 21, 2000-2024. 
Vrech, S.M., Etse, G., 2006. Geometrical localization analysis of gradient-dependent parabolic Drucker-Prager elastoplasticity. Int. J. Plasticity 22, 943-964.

Wu, P.D., Graf, A., MacEwen, S.R., Lloyd, D.J., Jain, M., Neale, K.W., 2005a. On forming limit stress diagram analysis. Int. J. Solids Struct. 42, 2225-2241.

Wu, P.D., Lloyd, D.J., Jain, M., Neale K.W., Huang, Y., 2007. Effects of spatial grain orientation distribution and initial surface topography on sheet metal necking. Int. J. Plasticity 23, 1084-1104.

Wu, P.D., MacEwen, S.R., Lloyd, D.J., Jain, M., Tugcu, P., Neale, K.W., 2005b. On pre-straining and the evolution of material anisotropy in sheet metals. Int. J. Plasticity 21, 723-739.

Yoshida, K., Ishizaka, T., Kuroda, M., Ikawa, S., 2007a. The effects of texture on formability of aluminum alloy sheets. Acta Materialia 55, 4499-4506.

Yoshida, K., Kuwabara, T., 2007. Effect of strain hardening behavior on forming limit stresses of steel tube subjected to nonproportional loading paths. Int. J. Plasticity 23, 1260-1284.

Yoshida, K., Kuwabara, T., Kuroda, M., 2007b. Path-dependence of the forming limit stresses in a sheet metal. Int. J. Plasticity 23, 361-384.

Yoshida, K., Suzuki, N., 2008. Forming limit stresses predicted by phenomenological plasticity theories with anisotropic work-hardening behavior. Int. J. Plasticity, 24, 118139. 
Zhang, F., Bower, A.F., Mishra, R.K., Boyle, K.P., 2008. Numerical simulations of necking during tensile deformation of aluminum single crystals. Int. J. Plasticity, doi:10.1016/j.ijplas.2007.12.006.

Zhou, Y., Neale, K.W., 1995. Predictions of forming limit diagrams using a ratesensitive crystal plasticity model. Int. J. Mech. Sci. 37, 1-20.

Willis, J., 1988. (Private communication). 


\section{ACCEPTED MANUSCRIPT}

Table 1. Slip system numbering for B.C.C. crystals

Slip system numbering for the family $<111>\left(\begin{array}{lll}1 & 1 & 0\end{array}\right)$

\begin{tabular}{ccccccccc}
\hline System & $\overrightarrow{\mathbf{m}}$ & $\overrightarrow{\mathbf{n}}$ & System & $\overrightarrow{\mathbf{m}}$ & $\overrightarrow{\mathbf{n}}$ & System & $\overrightarrow{\mathbf{m}}$ & $\overrightarrow{\mathbf{n}}$ \\
\hline 1 & $<111>$ & {$[1 \overline{1} 0]$} & 5 & $<\overline{1} 11>$ & {$[101]$} & 9 & $<1 \overline{1} 1>$ & {$[011]$} \\
2 & $<111>$ & {$[10 \overline{1}]$} & 6 & $<\overline{1} 11>$ & {$[01 \overline{1}]$} & 10 & $<11 \overline{1}>$ & {$[1 \overline{1} 0]$} \\
3 & $<111>$ & {$[01 \overline{1}]$} & 7 & $<1 \overline{1} 1>$ & {$[110]$} & 11 & $<11 \overline{1}>$ & {$[101]$} \\
4 & $<\overline{1} 11>$ & {$[110]$} & 8 & $<1 \overline{1} 1>$ & {$[10 \overline{1}]$} & 12 & $<11 \overline{1}>$ & {$[011]$} \\
\hline
\end{tabular}

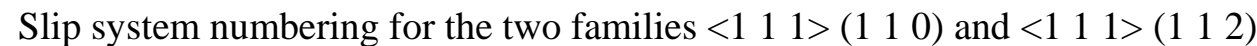

\begin{tabular}{ccccccccc}
\hline System & $\overrightarrow{\mathbf{m}}$ & $\overrightarrow{\mathbf{n}}$ & System & $\overrightarrow{\mathbf{m}}$ & $\overrightarrow{\mathbf{n}}$ & System & $\overrightarrow{\mathbf{m}}$ & $\overrightarrow{\mathbf{n}}$ \\
\hline 1 & $<111>$ & {$[1 \overline{1} 0]$} & 9 & $<\overline{1} 11>$ & {$[01 \overline{1}]$} & 17 & $<1 \overline{1} 1>$ & {$[121]$} \\
2 & $<111>$ & {$[10 \overline{1}]$} & 10 & $<\overline{1} 11>$ & {$[1 \overline{1} 2]$} & 18 & $<1 \overline{1} 1>$ & {$[21 \overline{1}]$} \\
3 & $<111>$ & {$[01 \overline{1}]$} & 11 & $<\overline{1} 11>$ & {$[12 \overline{1}]$} & 19 & $<11 \overline{1}>$ & {$[1 \overline{1} 0]$} \\
4 & $<111>$ & {$[11 \overline{2}]$} & 12 & $<\overline{1} 11>$ & {$[211]$} & 20 & $<11 \overline{1}>$ & {$[101]$} \\
5 & $<111>$ & {$[1 \overline{2} 1]$} & 13 & $<1 \overline{1} 1>$ & {$[110]$} & 21 & $<11 \overline{1}>$ & {$[011]$} \\
6 & $<111>$ & {$[211]$} & 14 & $<1 \overline{1} 1>$ & {$[10 \overline{1}]$} & 22 & $<11 \overline{1}>$ & {$[112]$} \\
7 & $<\overline{1} 11>$ & {$[110]$} & 15 & $<1 \overline{1} 1>$ & {$[011]$} & 23 & $<11 \overline{1}>$ & {$[\overline{1} 21]$} \\
8 & $<\overline{1} 11>$ & {$[101]$} & 16 & $<1 \overline{1} 1>$ & {$[\overline{1} 12]$} & 24 & $<11 \overline{1}>$ & {$[2 \overline{1} 1]$} \\
\hline
\end{tabular}




\section{ACCEPTED MANUSCRIPT}

Table 2. Comparison between the active slip systems found by the present model and those from the literature (Nesterova et al., 2001)

\begin{tabular}{cccccc}
\hline $\begin{array}{c}\text { Rolling } \\
\text { convention }\end{array}$ & $\begin{array}{c}\text { Major slip } \\
\text { systems } \\
\text { (Nesterova } \text { et } \\
\text { al.) }\end{array}$ & $\begin{array}{c}\text { Minor slip } \\
\text { systems } \\
\text { (Nesterova et } \\
\text { al.) }\end{array}$ & $\begin{array}{c}\text { Major slip } \\
\text { systems } \\
\text { (present } \\
\text { model) }\end{array}$ & $\begin{array}{c}\text { Minor slip } \\
\text { systems } \\
\text { (present } \\
\text { model) }\end{array}$ & $\begin{array}{c}\text { Residual slip } \\
\text { systems } \\
\text { (present } \\
\text { model) }\end{array}$ \\
\hline$(111)[1 \overline{2} 1]$ & 8 & 2 & 8 & 2 & $1,4,6,7,9,10,12$ \\
$(111)[1 \overline{1} 0]$ & 10 & 1 & 10 & 1 & $3,5,6,8,11,12$ \\
$(111)[14 \overline{15}]$ & 10,8 & 1,2 & 10,8 & 1,2 & $3,5,6,7,12$ \\
$(111)[11511]$ & 6,8 & 3,11 & 6,8 & 3 & $4,7,9$ \\
$(001)[100]$ & $2,3,5,6,8,9$, & None & $2,3,5,6,8,9$, & None & None \\
\hline
\end{tabular}


Table 3. Identified parameters for the two steels

\begin{tabular}{lllll}
\hline Parameter & $\mathrm{D}$ & $\tau_{c 0}$ & $y_{c}$ & $g_{0}$ \\
\hline Single Phase & $20.10^{-6} \mathrm{~m}$ & $55 \mathrm{MPa}$ & $3.25 \mathrm{~nm}$ & 100 \\
Dual Phase: Phase 1 & $5.10^{-6} \mathrm{~m}$ & $122 \mathrm{MPa}$ & $2.4 \mathrm{~nm}$ & 140 \\
Dual Phase: Phase 2 & $1.310^{-6} \mathrm{~m}$ & $550 \mathrm{MPa}$ & $2.4 \mathrm{~nm}$ & 120 \\
\hline
\end{tabular}


Fig. 1. Effect of parameter $\mathrm{k}$ - Parametric study of the smooth approximation to the

Heaviside step function: the regularizing role of the logistic function is illustrated (higher values of k correspond to sharper transition).

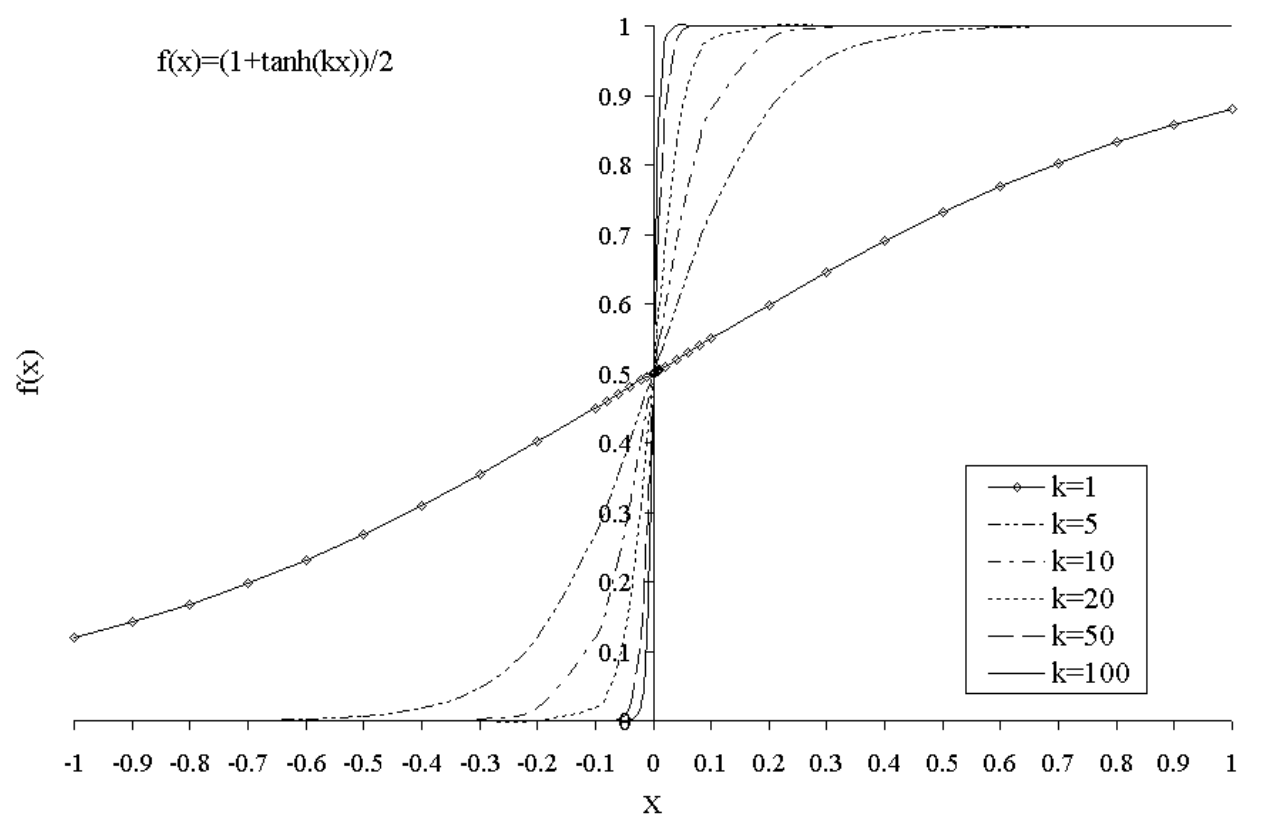


Fig. 2. Simulation of a loading-unloading-reloading uniaxial tensile test.

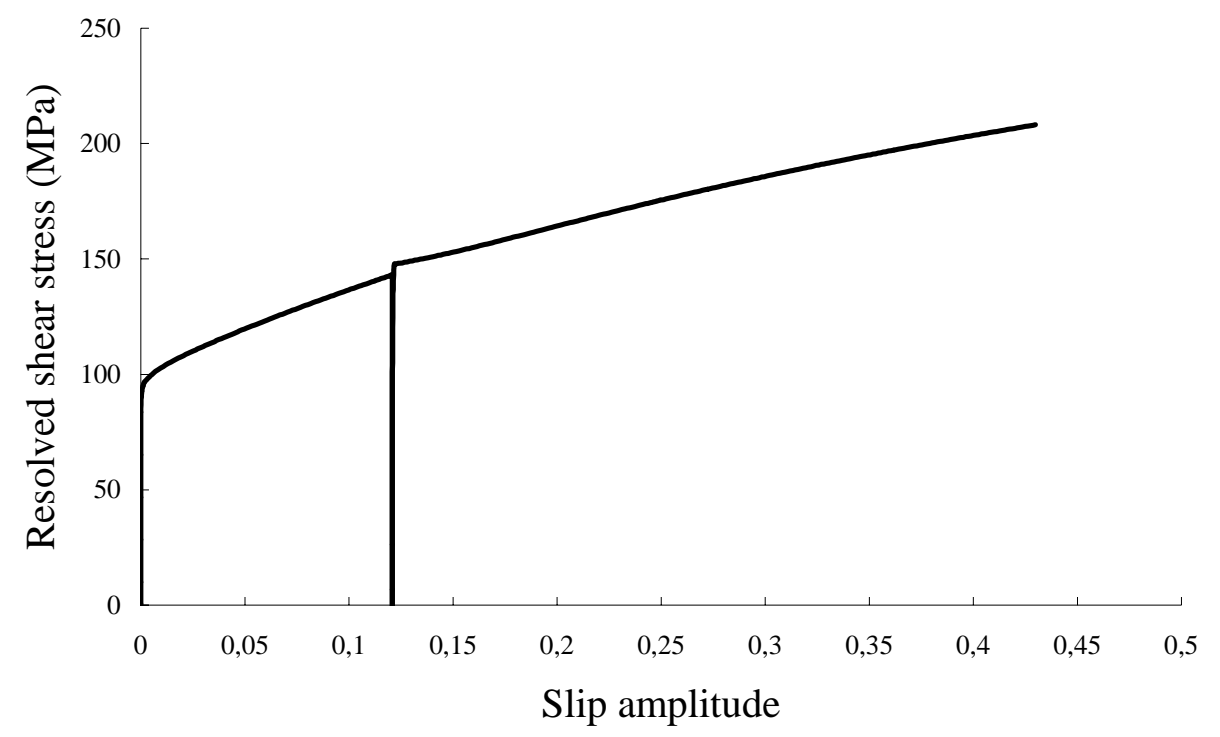


Fig. 3. Effect of orientation terms on the single crystal behavior.

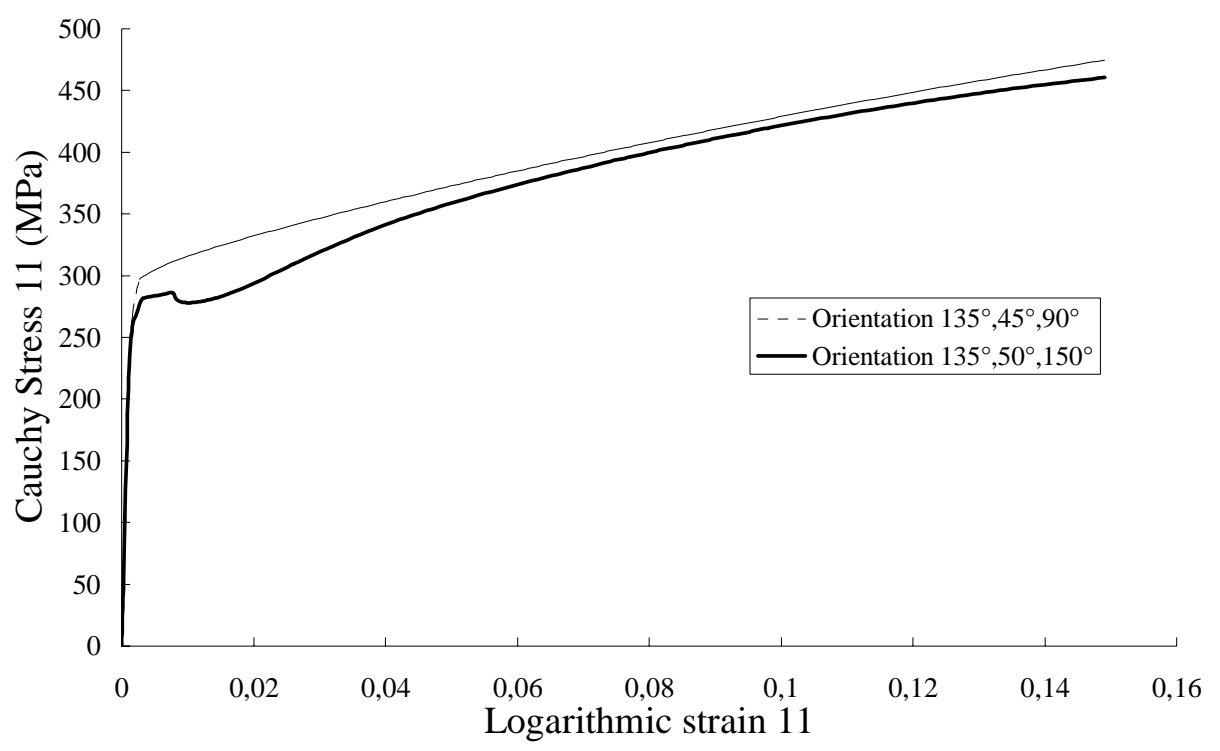


Fig. 4. Evolution of Euler's angles for two different single crystals.

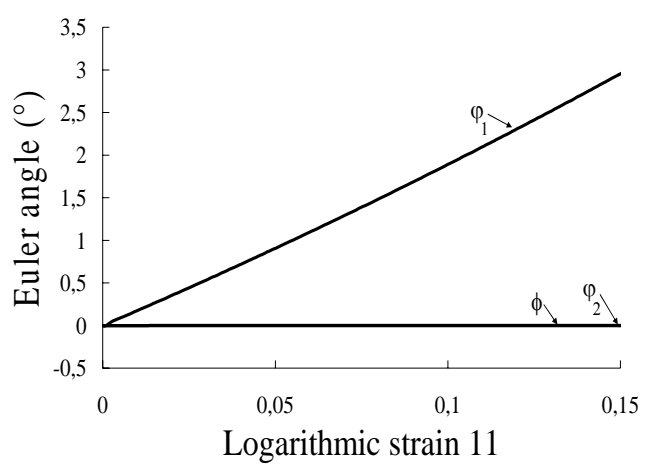

Orientation $135^{\circ}, 45^{\circ}, 90^{\circ}$

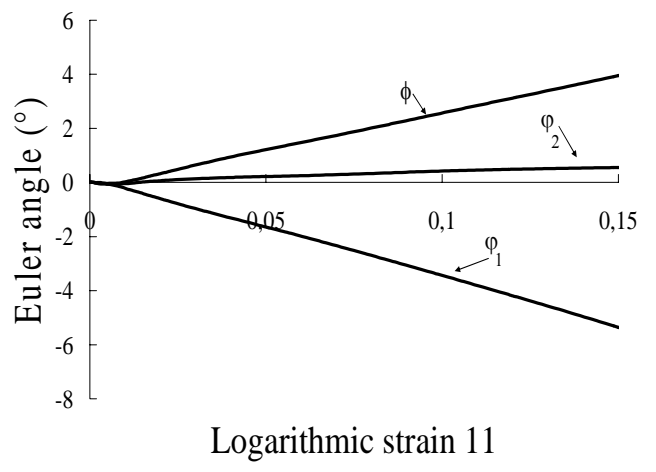

Orientation $135^{\circ}, 50^{\circ}, 150^{\circ}$ 
Fig. 5. Schematic representation defining the three Euler angles $\varphi_{1}, \phi, \varphi_{2}$ according to Bunge’s notation.

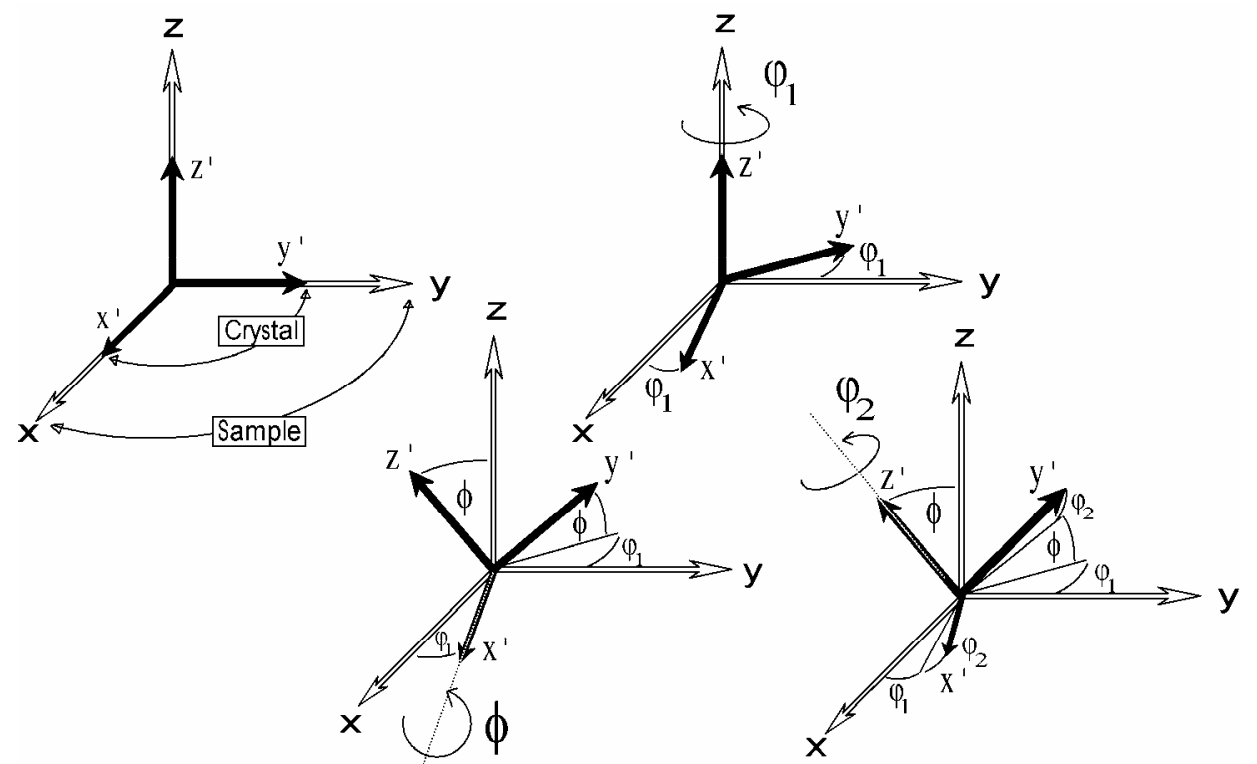


Fig. 6. Comparison between simulations and experiments for a single phase steel.

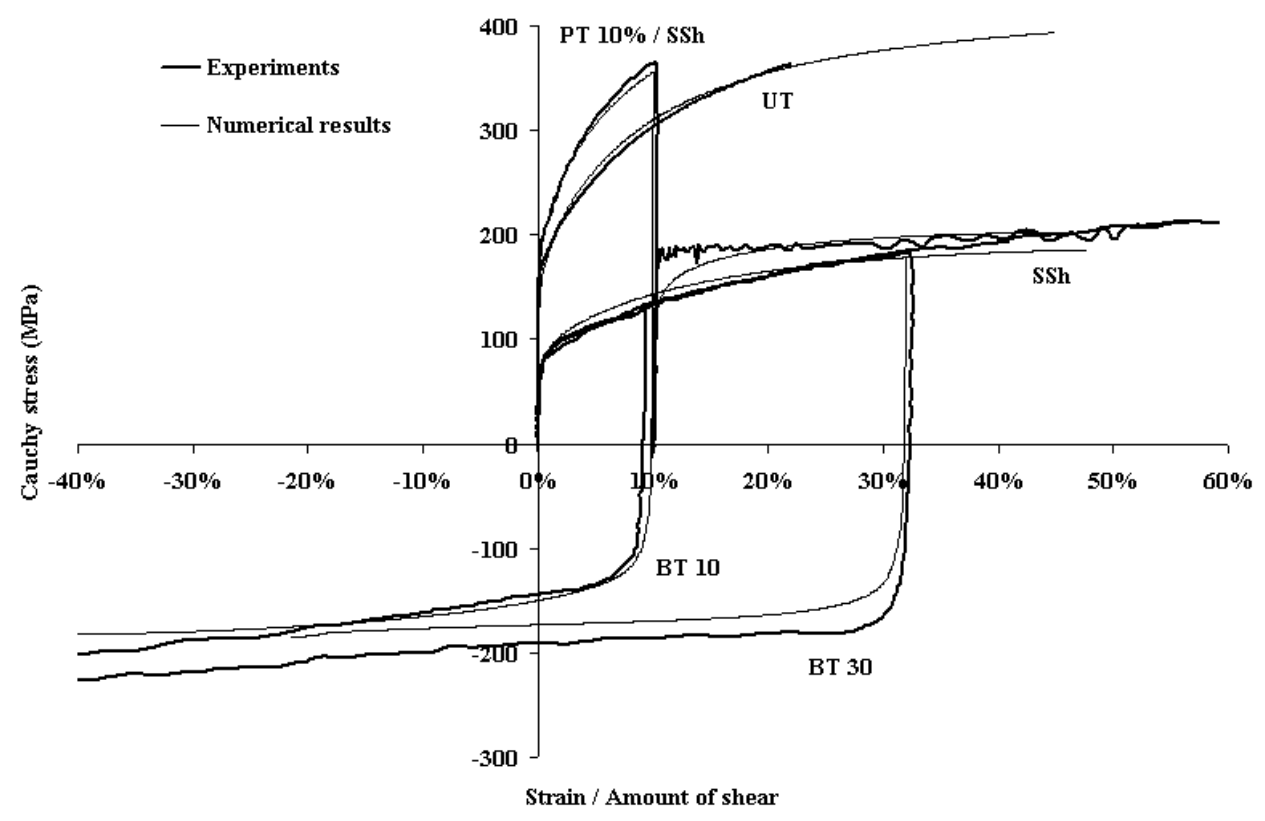

UT stands for Uniaxial Tension, BT for Bauschinger Test, SSh for Simple Shear and PT $10 \%$ SSh for Plane Tension until 10\% of logarithmic strain followed by Simple Shear. 
Fig. 7. Comparison between simulations and experiments for a dual-phase steel.

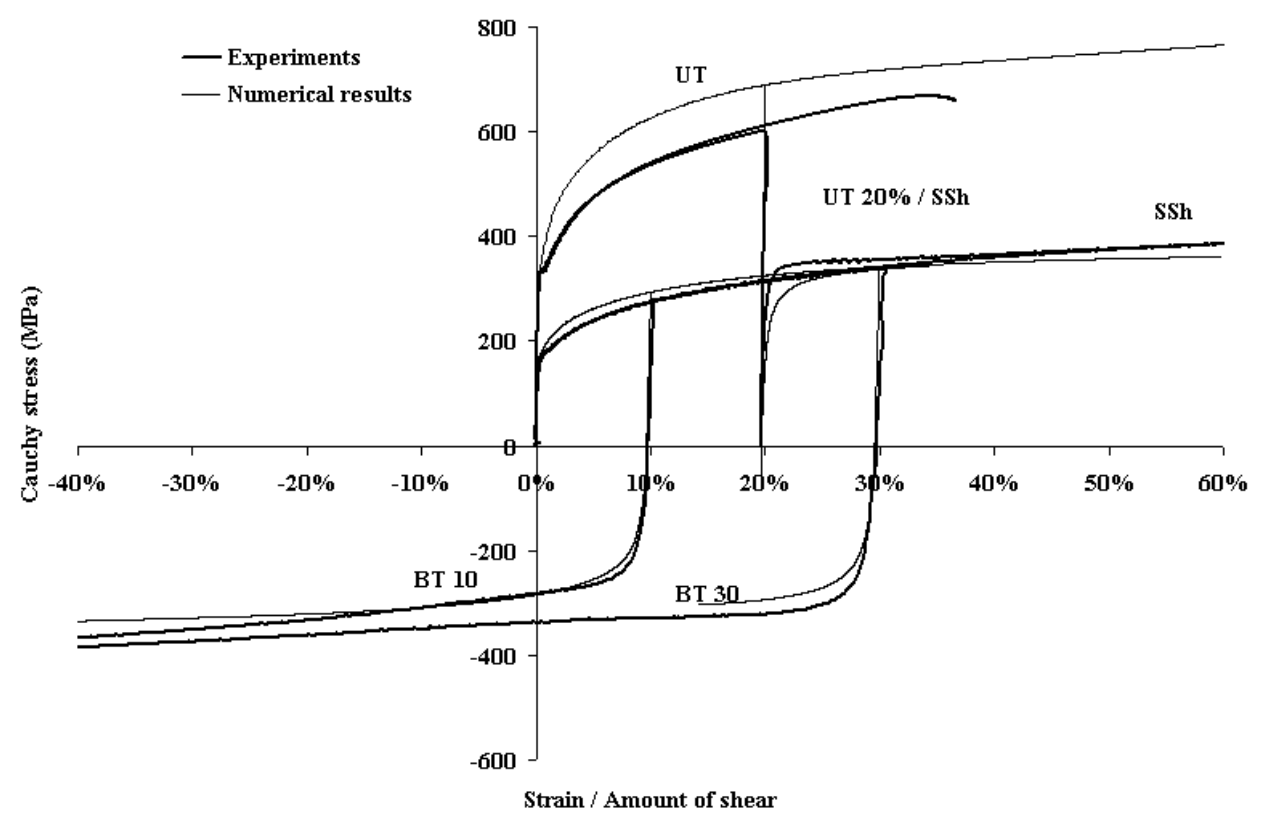

UT stands for Uniaxial Tension, BT for Bauschinger Test, SSh for Simple Shear and UT $20 \%$ SSh for Uniaxial Tension until $20 \%$ of logarithmic strain followed by Simple Shear. 
Fig. 8. Localization of the deformation along a shear band.

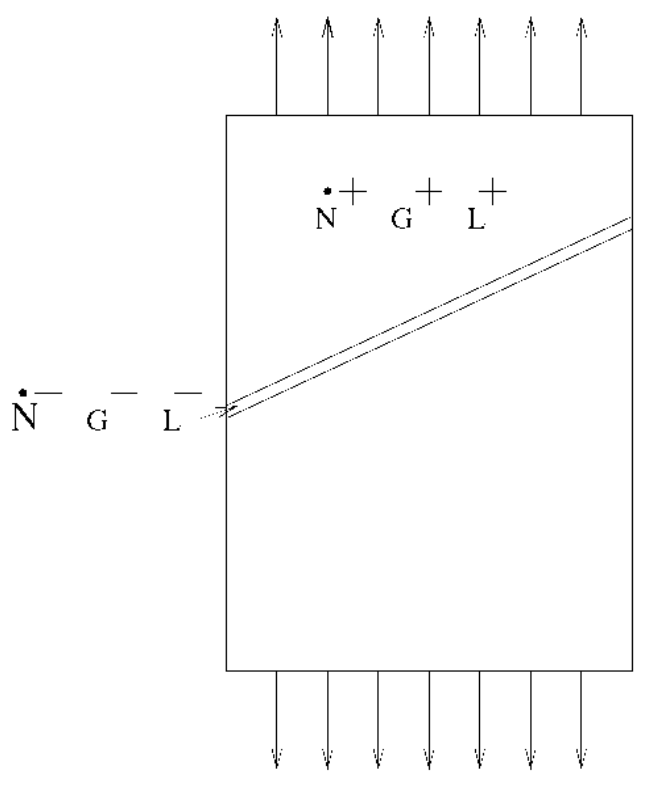


Fig. 9. Volume fraction of localized grains during a tensile test.

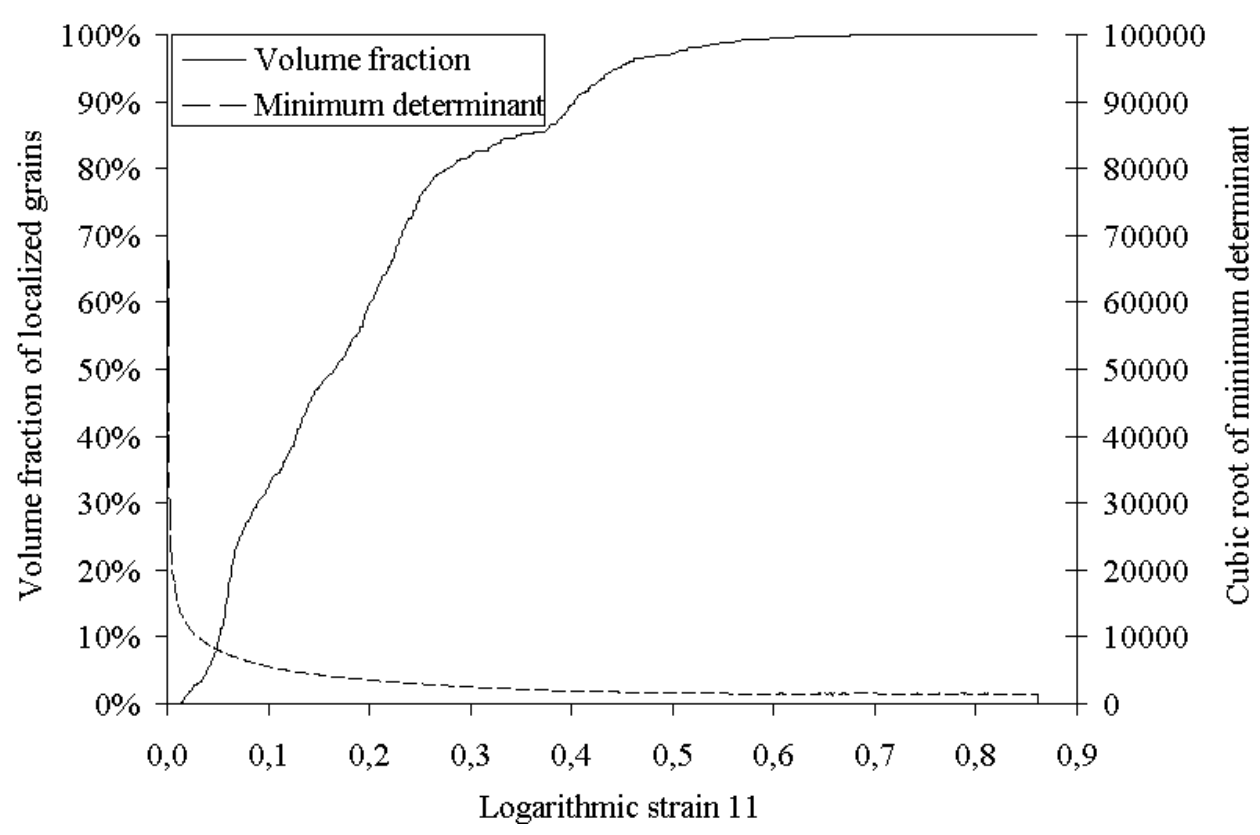


Fig. 10. Validation of ArcelorMittal's FLD model on several grades of steel for linear loading paths.

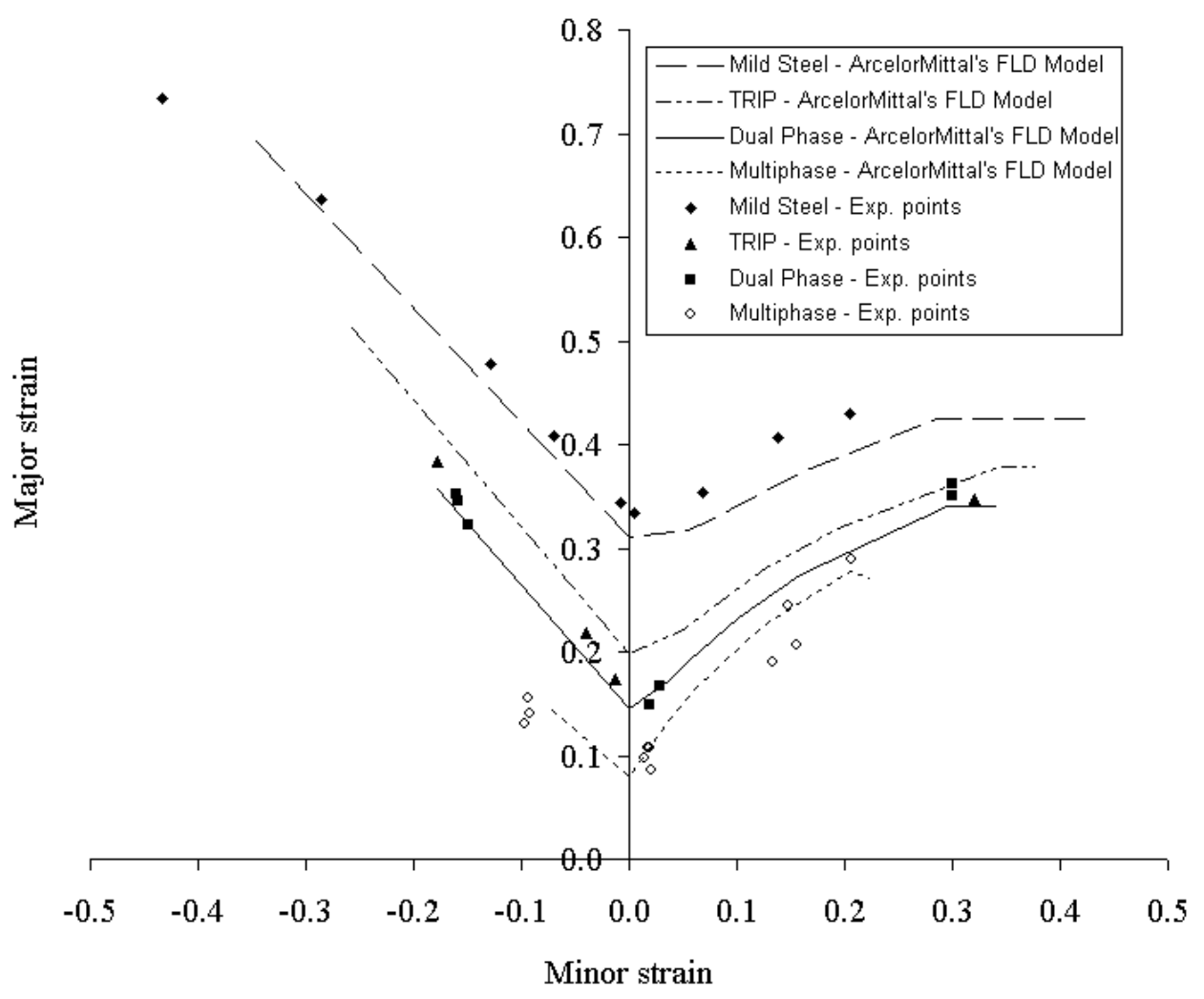


Fig. 11. Ellipticity loss prediction: comparison between direct ELD and ArcelorMittal's reference FLD for a single phase steel.

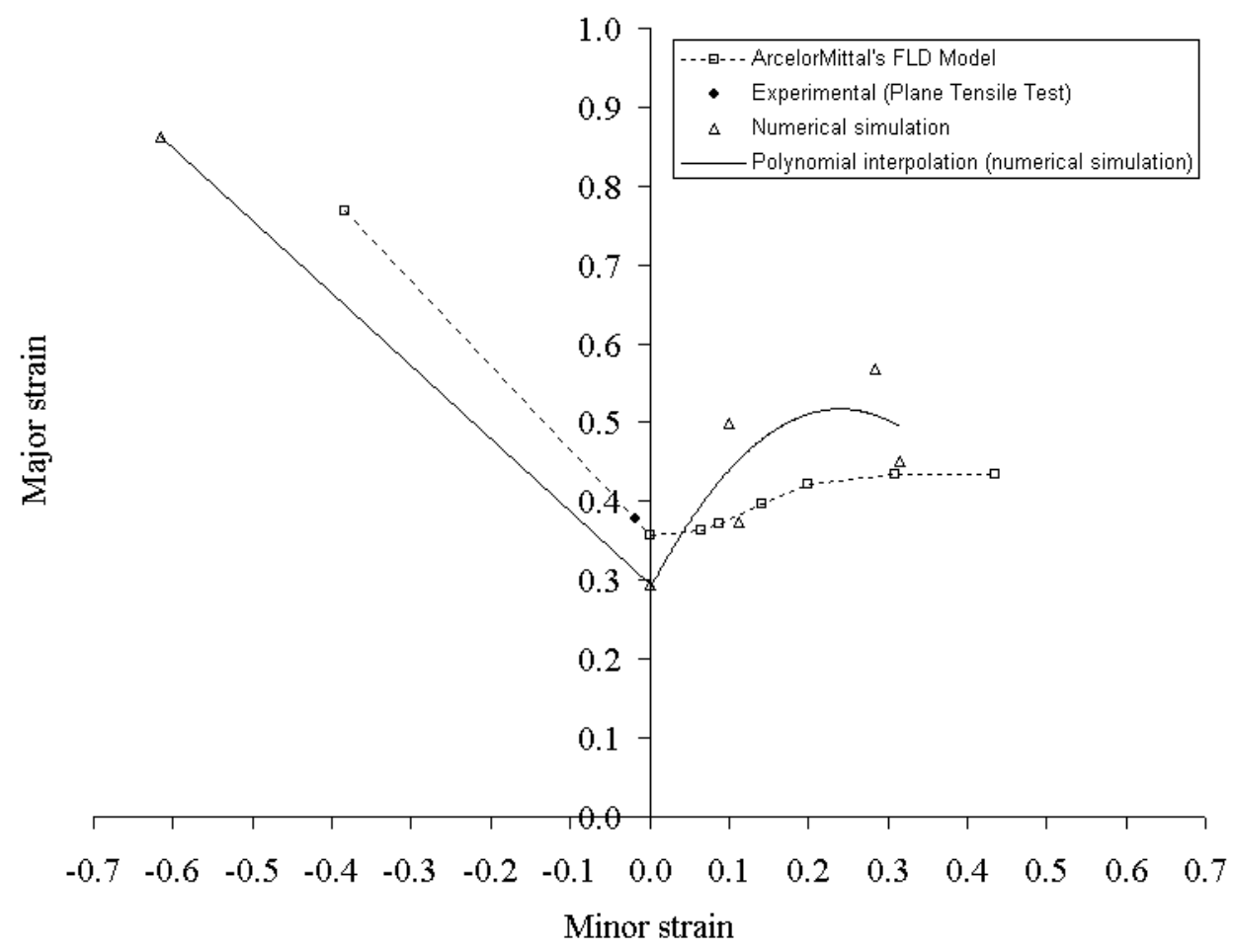


Fig. 12. Ellipticity loss prediction: comparison between direct ELD and ArcelorMittal's reference FLD for a dual-phase steel.

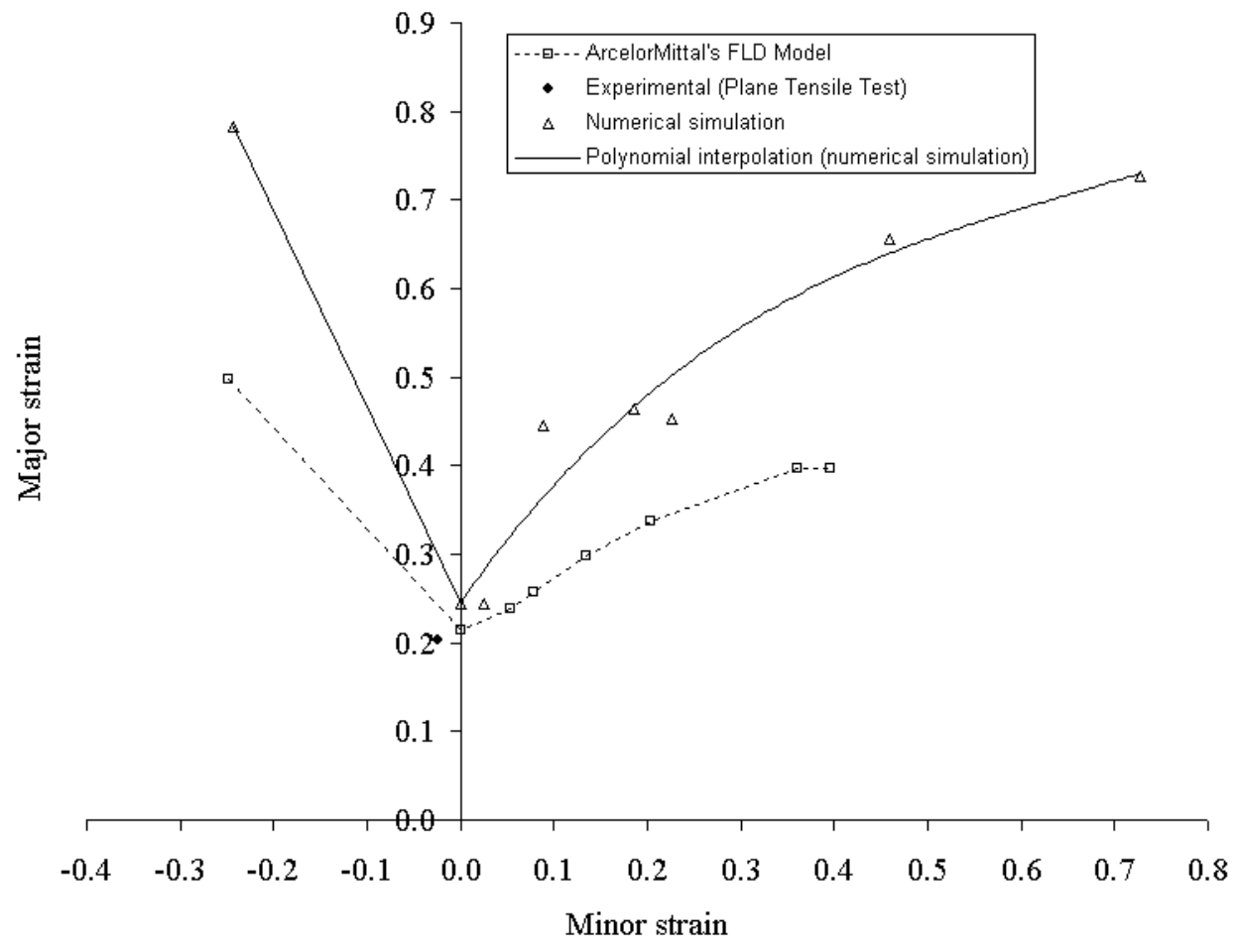


Fig. 13. Ellipticity loss simulations (ELDs) for a single phase steel for linear and complex loading paths.

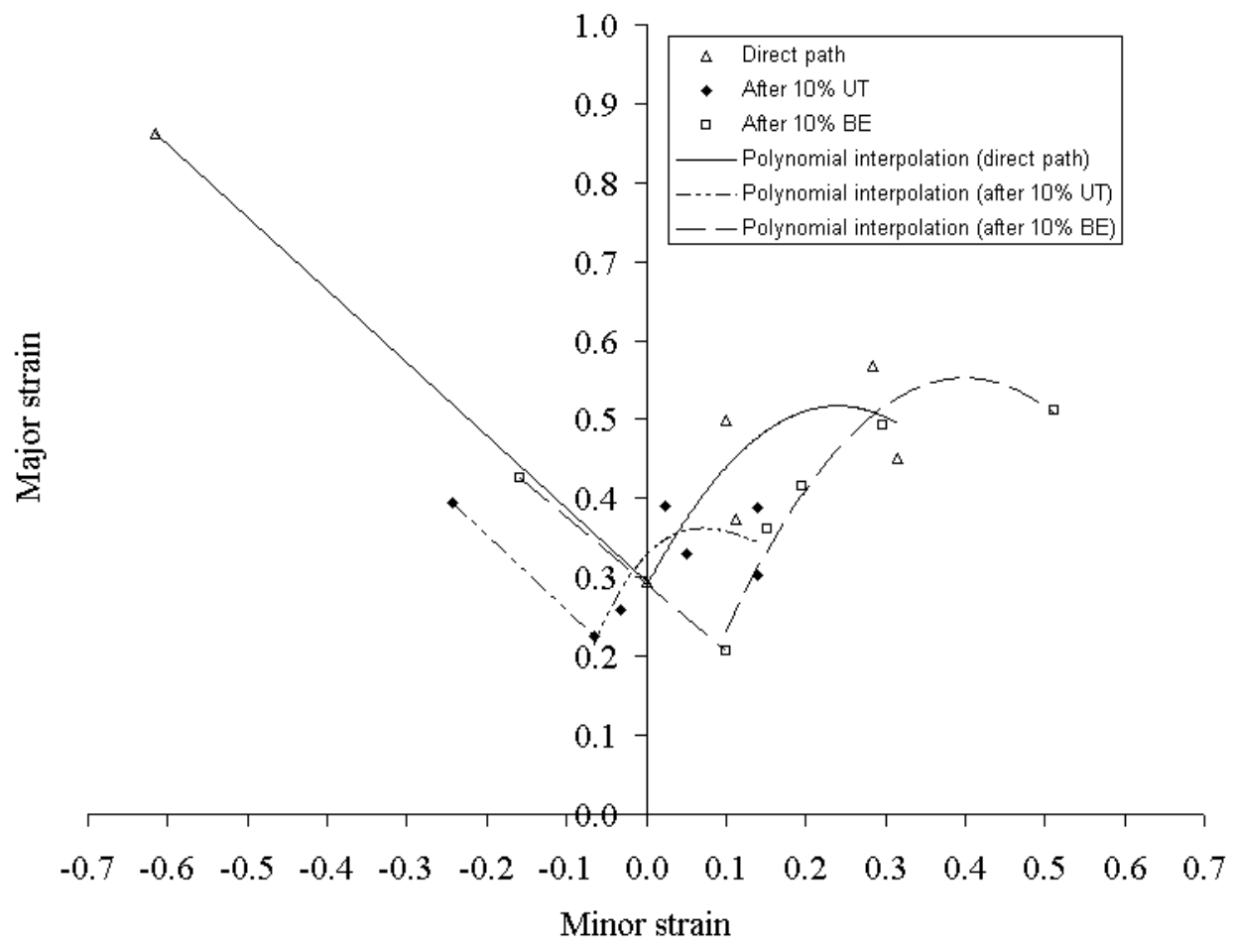

UT stands for Uniaxial Tension pre-strain, BE for Biaxial Expansion pre-strain. 
Fig. 14. Ellipticity loss simulations (ELDs) for a dual-phase steel for linear and complex loading paths.

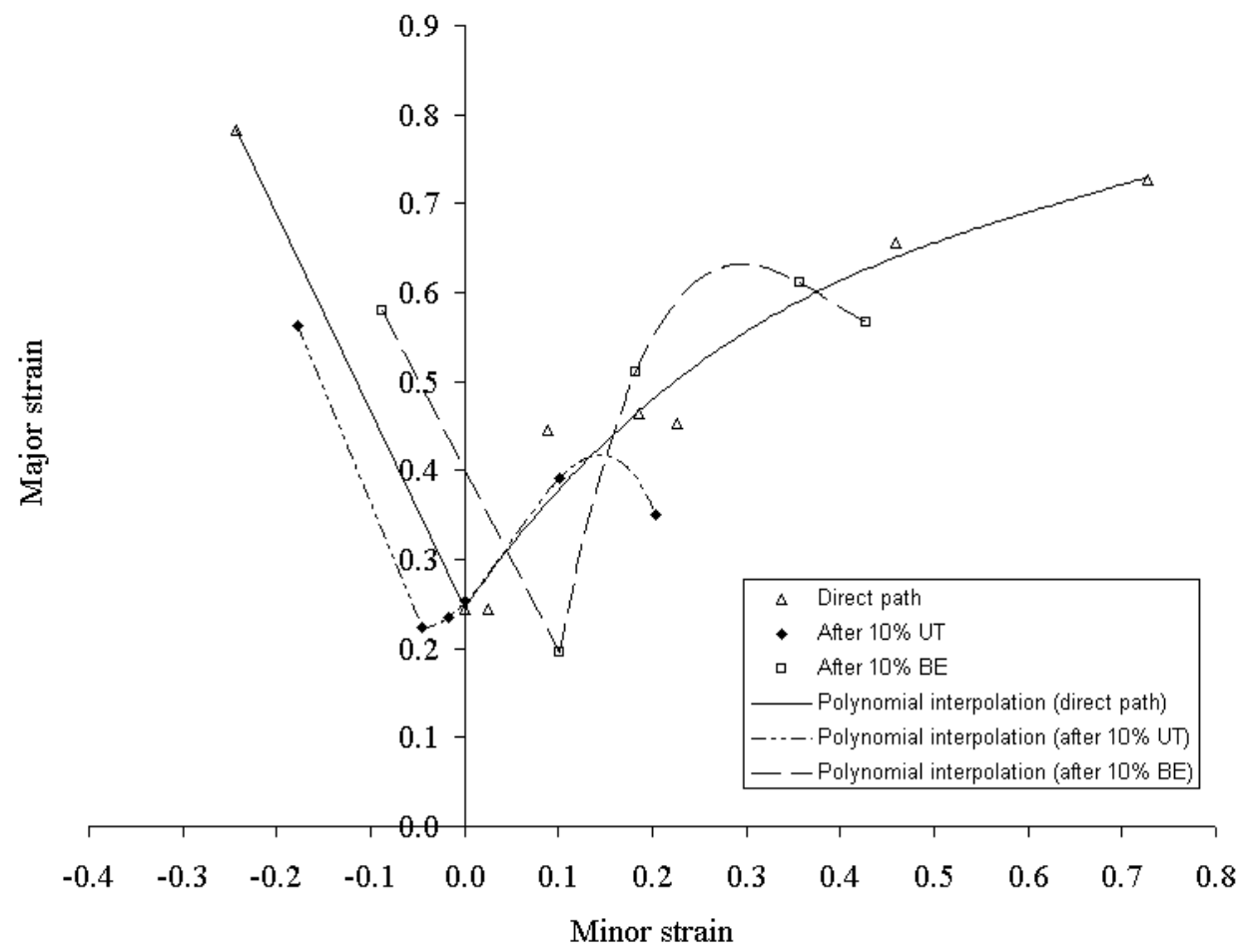

UT stands for Uniaxial Tension pre-strain, BE for Biaxial Expansion pre-strain. 
Fig. 15. Softening effect due to dislocation annihilation - impact on ductility during a uniaxial tensile test for a single-phase steel.

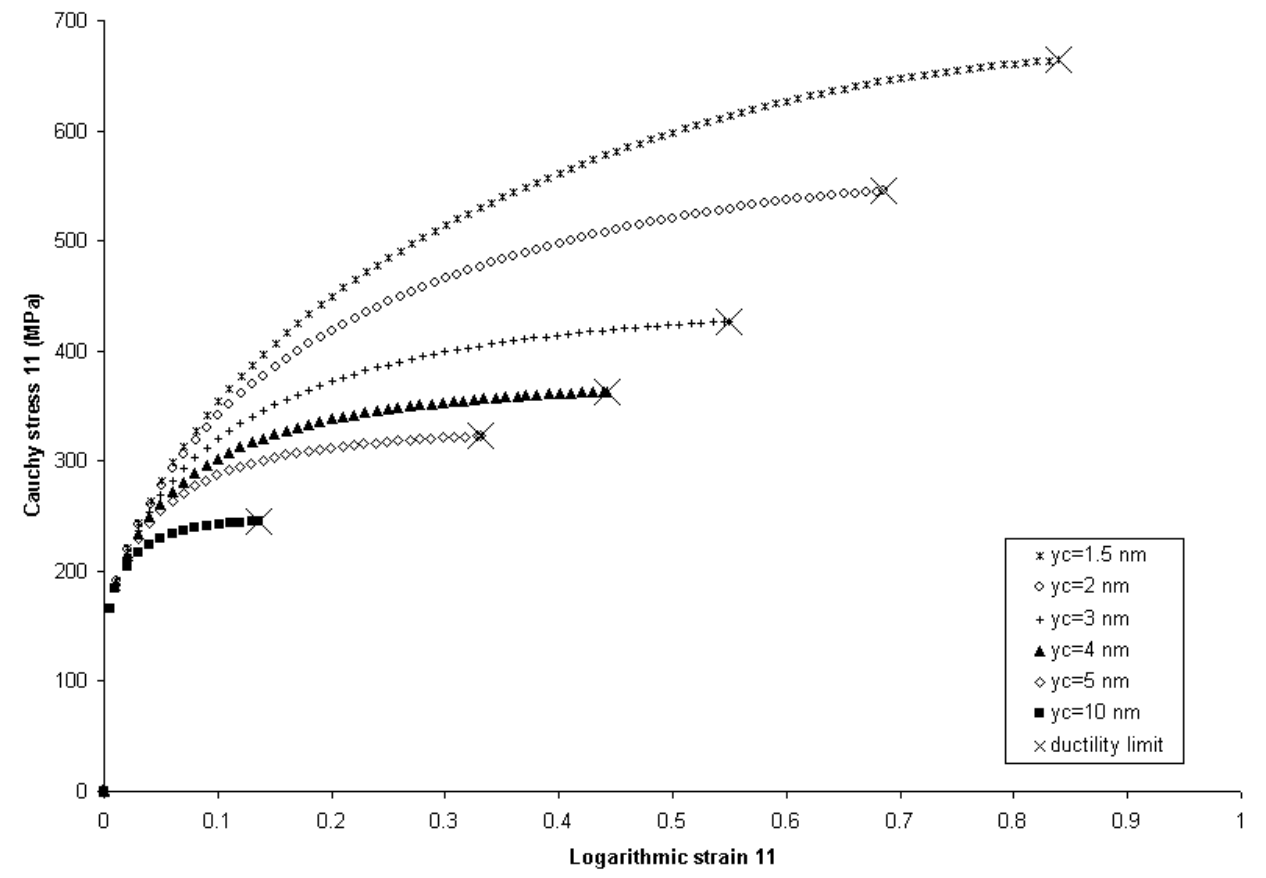


Fig 16. In-plane and out-of-plane orientation of the localization band of the sheet.

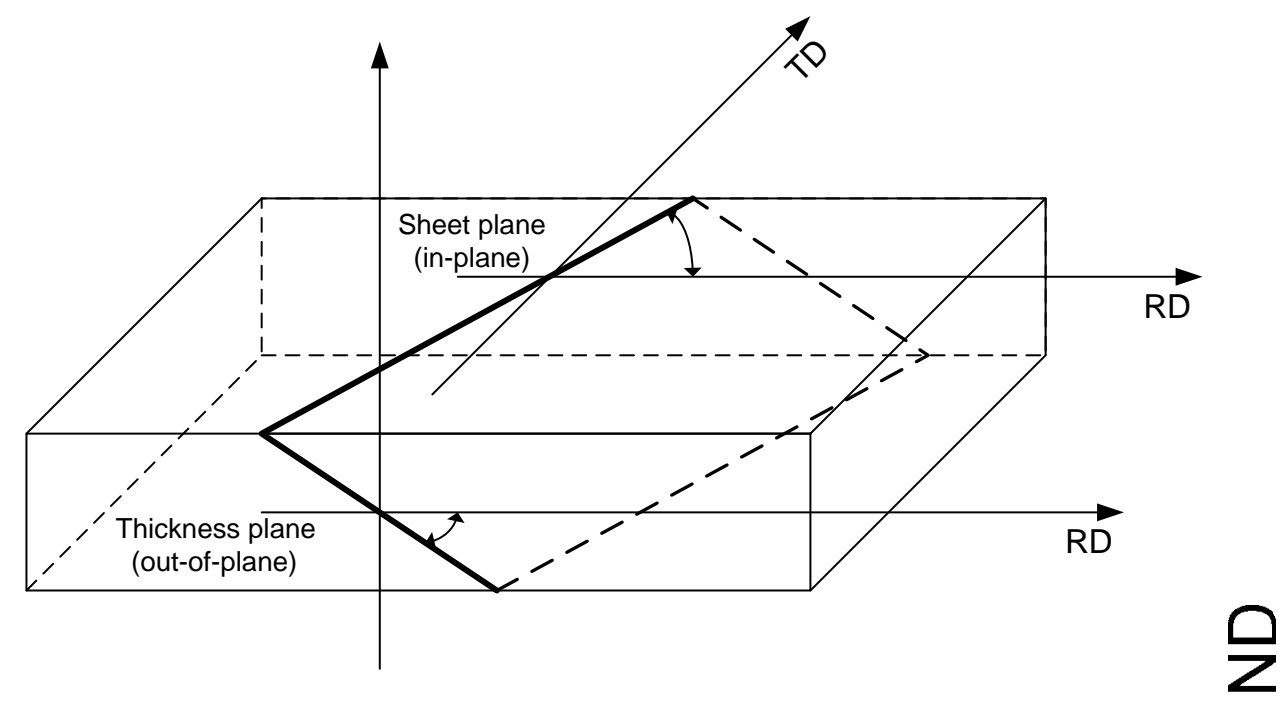


Table 4. In-plane and out-of-plane orientation of the localization band of the sheet for the two steels for two different loading modes

\begin{tabular}{cccccc}
\hline & Steel & \multicolumn{2}{c}{ Single Phase } & \multicolumn{2}{c}{ Dual Phase } \\
\cline { 2 - 6 } & Orientation & $\theta_{1}$ & $\theta_{2}$ & $\theta_{1}$ & $\theta_{2}$ \\
\hline Loading & Uniaxial Tension & $52^{\circ}$ & $90^{\circ}$ & $51^{\circ}$ & $90^{\circ}$ \\
mode & Plane Tension & $90^{\circ}$ & $46^{\circ}$ & $90^{\circ}$ & $46^{\circ}$ \\
\hline
\end{tabular}

